\title{
Self-consistent theory of shot noise in nondegenerate ballistic conductors
}

\author{
O. M. Bulashenko and J. M. Rubí \\ Departament de Física Fonamental, Universitat de Barcelona, Diagonal 647, E-08028 Barcelona, Spain \\ V. A. Kochelap \\ Department of Theoretical Physics, Institute of Semiconductor Physics, Kiev 252028, Ukraine
}

(Received 12 August 1999)

\begin{abstract}
A self-consistent theory of shot noise in ballistic two-terminal conductors under the action of long-range Coulomb correlations is presented. Analytical formulas for the electron distribution function and its fluctuation along the conductor, which account for the Coulomb correlations, have been derived. Based upon these formulas, the current-noise reduction factor has been obtained for biases ranging from thermal to shot-noise limits as dependent on two parameters: the ratio between the length of the sample and the Debye screening length $\lambda=d / L_{D}$ and the applied voltage $q U / k_{B} T$. The difference with the formulas for a vacuum diode is discussed.
\end{abstract}

\section{INTRODUCTION}

Recently, significant attention has been focused on the study of nonequilibrium fluctuations of current (shot noise) in mesoscopic conductors. ${ }^{1}$ The term "shot noise," appearing originally in the context of pure ballistic electron transmission in vacuum-tube devices, ${ }^{2}$ has acquired nowadays a much broader usage and refers to different mesoscopic structures, including diffusive conductors, and resonant-tunneling devices, where the carrier flow exhibits nonequilibrium noise proportional to the electric current. ${ }^{1}$

A matter of particular interest is the significance of longrange Coulomb correlations in the noise-reduction effect. ${ }^{3,4}$ Coulomb interactions may keep nearby electrons apart and more regularly spaced rather than strictly at random, which leads to the noise reduction, as pointed out by Landauer. ${ }^{3}$ This effect occurs in different physical situations. Among them are charge-limited ballistic transport, resonant tunneling, single-electron tunneling, etc. For the ballistic conductors an electrostatic potential barrier is formed near an injecting contact. The barrier fluctuates synchronously with random electron passages through it, which leads to noise reduction, as evidenced recently by Monte Carlo simulations for semiconductor ballistic diodes. ${ }^{5}$ In this way, an incoming Poissonian flow is converted into an outgoing sub-Poissonian flow, exhibiting a motional electron-number squeezing. ${ }^{6}$ This effect is similar to that leading to shot-noise suppression in vacuum diodes. ${ }^{7-9}$ Under the resonant tunneling effect, a built-in charge inside a quantum well affects the position of the resonant level and prevents the incoming carriers from passing through the well, thereby resulting in carrier correlation and shot-noise reduction ${ }^{10-12}$ in a certain range of biases. ${ }^{13,14}$ The Coulomb correlations in these systems act under the coherent as well as under the sequential tunneling regime of the carrier transport. The carrier correlations reach their extreme form of the Coulomb blockade of the electron transfer under the single-electron tunneling effect, leading to the noise reduction studied theoretically ${ }^{15-20}$ and observed in experiment. $^{21}$

All the above-mentioned cases have the common features that are necessary for the Coulomb regulation effect and shot-noise reduction in the whole frequency spectrum to occur: (i) the existence of a potential barrier inside a device or at the interface with an injecting electron reservoir, which controls the current; (ii) the dependence of the barrier height and/or carrier transmission on the current. If no barrier is present, no shot-noise reduction at low frequencies due to Coulomb repulsion is expected. At high frequencies, however, the noise level may also be affected by Coulomb correlations due to screening in an external environment. ${ }^{22,23}$

The potential barrier, which controls the current, appears in an ordinary situation of the space-charge-limited transport. For ballistic nondegenerate conductors this case has been treated recently by Monte Carlo simulations ${ }^{5,6}$ and attracted some attention in Ref. 24 for degenerate case. For the case of diffusive nondegenerate conductors, studied by the Monte Carlo technique in Ref. 25, the self-consistent kinetic theory of noise, which takes into account Coulomb correlations, has been developed recently in Refs. 26 and 27. A similar kinetic theory for the ballistic case is lacking.

It is the aim of this paper to address the problem of Coulomb correlations in ballistic conductors and present a selfconsistent theory of shot noise in these conductors by solving analytically the kinetic equation coupled self-consistently with the Poisson equation. It is important to compare the present noise theory for a semiconductor ballistic diode with that for a vacuum diode developed long ago. ${ }^{8,9}$ The main advance for the latter has been done in the celebrated paper by North published in 1940, where he derived an asymptotic formula for the current-noise spectral density at the high voltage limit. ${ }^{8}$ Monte Carlo simulations of noise in vacuum diodes are also available. ${ }^{28-30}$ It should be stressed, however, that despite the similarity of the underlying physics (in both cases the nondegenerate Boltzmann electron gas without collisions in the electrostatic field is under consideration), the case of the semiconductor diode differs by several features: (i) due to a two-terminal geometry, there are two opposing currents instead of a single current, which results in different current-voltage characteristics at low and moderate biases, ${ }^{31}$ (ii) the ballistic transport regime is limited by the presence of 
disorder, impurities, etc. Even in a pure and perfect solid, carriers may interact with a lattice (phonons), which at high biases becomes significant and breaks down the ballistic regime. This makes it practically impossible to attain in solids the regime where the known formulas for vacuum electronics, such as the Child law for $I-V$ characteristics or North's asymptotic formula for the noise, may be applied. This issue will be addressed in the paper, using the derived formulas and considering them in a full range of biases. Finally, we suggest an electron spectroscopy experiment to make the Coulomb correlations effect observable. The possibility of such an experiment is based on recent advances in nanoscale fabrication techniques and shot noise measurements. ${ }^{34-36}$

The paper is organized as follows. In Sec. II we describe the semiconductor ballistic structure and discuss the main assumptions concerning underlying physics. In particular, the validity of the one-dimensional plane geometry approximation for the fluctuation problem is addressed. In Sec. III we introduce the basic equations that describe the spacecharge-limited semiclassical transport: the collisionless kinetic equation coupled self-consistently with the Poisson equation. The steady-state problem is solved in Sec. IV, and the results are compared with the Monte Carlo simulations. In Sec. V we solve analytically the fluctuation problem and derive the formula for the current-noise spectral density that covers the range of biases from thermal to the shot-noise limits. The results for the noise-reduction factor are compared with Monte Carlo simulations and North's asymptotic formula for vacuum diodes. The contributions of different electron energy groups to the noise are found, and the correlations in energies for the electrons collected at the receiving contact are discussed. Finally, Sec. VI summarizes the main contributions of the paper, and in the Appendix we present mathematical details concerning the derivation of the fluctuations of the electron distribution function in the selfconsistent electric field.

\section{THE PHYSICAL MODEL}

Before proceeding with a discussion of the problem, we will specify the structure under consideration and the main assumptions concerning the underlying physics. Consider a two-terminal semiconductor ballistic sample with plane parallel contacts at $X=0$ and $X=d$ (see Fig. 1). The contacts, which we denote by $L$ and $R$ (left and right), are assumed to be heavily doped semiconductors with a contact electron density much higher than that in the sample. The structure may then be considered as a $n-i-n$ diode operating under a space-charge-limited current regime in which the current is determined by a charge injection from the contacts rather than by intrinsic carriers of the active region. ${ }^{37}$ Two different types of the contacts may be considered depending on whether the contact and the sample are fabricated of the same or different material. For the former case the diode is composed of two homojunctions [Fig. 1(a)], while for the latter, it is composed of two heterojunctions with a jump of the conductance band $\varepsilon_{c}$ at the contact-sample interface [Fig. 1(b)]. The underlying physics is similar if in both cases the contact doping is such that the Fermi level $\varepsilon_{F}$ is sufficiently below the edge of the conduction band in the sample. In such a case, only the tail of the distribution function is injected,

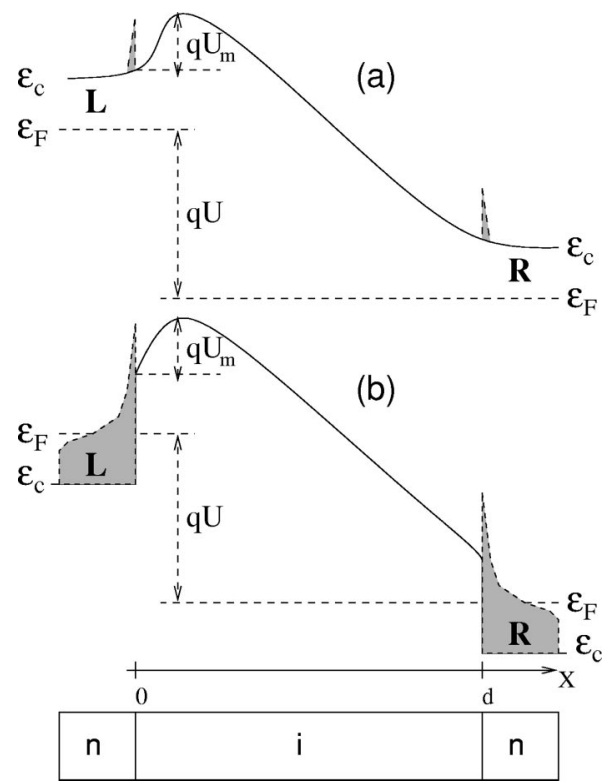

FIG. 1. Schematic band-energy diagram for a $n-i-n$ ballistic diode under a space-charge-limited conduction. Two different types of the contacts are shown: (a) homojunctions; (b) heterojunctions. Shadowed regions illustrate the energy distribution function of electrons at the contact-sample interfaces.

which leads to the nondegeneracy of the electron gas in the ballistic part of the diode. The theory is therefore applicable to quantum heterostructures with over-barrier transport, ${ }^{38}$ where current is determined by a tail in the distribution function (ballistic-injection, real-space-transfer devices, etc.), as well as for the homodiode with a nondegenerate electron gas in the contacts.

In order to simplify the problem, we assume that under the range of biases of interest, due to the large difference in the carrier density between the contacts and the sample, and hence in the corresponding Debye screening lengths, all the band bending occurs in the ballistic base, and therefore the relative position of the conduction band and the Fermi level $\varepsilon_{c}-\varepsilon_{F}$ does not change in the contacts. For such a modeling, all of the potential drop takes place exclusively inside the ballistic base between the positions $X=0$ and $X=d$ in Fig. 1, and the contacts may be excluded from the consideration. This assumption is better fulfilled for the case of the heterojunctions because of much higher electron densities in the contacts.

The carriers inside the contacts are assumed to remain at thermal equilibrium, and their injected part is distributed over the energy according to the Maxwell-Boltzmann distribution function at lattice temperature $T$. For the ballistic part of the diode, we suppose

$$
\lambda_{w} \ll d \leqslant \lambda_{p},
$$

with $\lambda_{w}$ the electron wavelength and $\lambda_{p}$ the mean free path, so that electrons may be considered as classical particles moving ballistically between the contacts and interacting with each other electrostatically. This regime is accessible in modern device fabrication technologies for which the mean free path $\lambda_{p}$ may be as high as $10^{4}-10^{5} \mathrm{~nm}$ in modulation- 
doped structures (for instance, in $\mathrm{GaAs} / \mathrm{Al}_{x} \mathrm{Ga}_{1-x} \mathrm{As}$ at low temperatures ${ }^{39,40}$ ) and $\sim 10^{3} \mathrm{~nm}$ in the purest bulk material, whereas the Fermi wavelength is about $40 \mathrm{~nm}$.

Next we assume that the transversal size of the diode is sufficiently thick (much larger than the screening length $L_{D}$ ). This allows us to treat the steady-state electrostatic problem as a one-dimensional one in the plane geometry. However, to use the same one-dimensional consideration for the fluctuation problem, we need an additional justification. The fluctuating current is determined by a random transmission of discrete electron charges of the amount of $q$. Essentially, this discreteness of charge transmission together with randomness leads to the shot noise. In principle, each single electron while transmitted between the contacts disturbs the electric field and thereby interacts with other electrons of the current flow in both longitudinal and transversal directions. The electrostatic screening in such a problem is threedimensional. Nevertheless, we shall treat the problem as a one-dimensional one considered in the plane geometry by averaging the fluctuations over the transversal directions. This is justified if the average distance between the excess (fluctuating) carriers in transversal direction is much smaller than the characteristic scale of the electrostatic potential variation in that direction. This condition may be written as

$$
L_{\perp}^{2} d \sqrt{\left\langle\delta n^{2}\right\rangle} \gg 1
$$

where $L_{\perp}$ is the transverse characteristic scale, $n$ is the typical electron density in the ballistic region, and $\delta n$ its fluctuation. To estimate the order of magnitude of the fluctuation $\delta n$, we use Poissonian statistics, leading to the relation $\left\langle\delta n^{2}\right\rangle \sim n /\left(L_{\perp}^{2} d\right)$. Thus, the condition (2) becomes $n L_{\perp}^{2} d$ $\gg 1$. The scale $L_{\perp}$ depends on the ratio between the longitudinal dimension $d$ of the sample and the Debye screening length $L_{D}$ in the active region. For nondegenerate electrons, the latter is defined as $L_{D}=\sqrt{\kappa k_{B} T /\left(q^{2} n\right)}$, with $\kappa$ being the dielectric permittivity and $k_{B}$ the Boltzmann constant. To estimate the magnitude of $L_{\perp}$, we distinguish two different cases: (i) Weak screening, $d \leqq L_{D}$ : For this case $L_{\perp} \sim d$, ${ }^{41}$ and condition (2) becomes $n \gg d^{-3}$, which for $d \sim 300 \mathrm{~nm}$ requires $n \gg 10^{14} \mathrm{~cm}^{-3}$. (ii) Strong screening, $d \gg L_{D}$ : For this case $L_{\perp} \sim L_{D}$, and condition (2) becomes $n \gg L_{D}^{-2} d^{-1}$. After the substitution of the expression for the screening length, it is seen that this condition becomes independent of $n$, although it requires a sufficiently long sample, $d$ $\gg q^{2} /\left(\kappa k_{B} T\right) \sim 2 a_{0}\left(E_{0} / k_{B} T\right)$, where $a_{0}=\kappa \hbar^{2} /\left(m q^{2}\right)$ is the effective Bohr radius and $E_{0}=q^{2} /\left(2 \kappa a_{0}\right)$ is the effective Rydberg energy in the material. For GaAs, $a_{0}$ $\approx 10 \mathrm{~nm}, E_{0} \approx 5 \mathrm{meV}$, which corresponds to the temperature of about $60 \mathrm{~K}$. Then for $T \sim 10 \mathrm{~K}, d \gg 120 \mathrm{~nm}$, which is supposed to be fulfilled. On another hand, the condition of strong screening requires $d \gg L_{D}$, which leads to the condition on the electron density

$$
n \gg \frac{k_{B} T}{E_{0}} \frac{1}{2 a_{0} d^{2}} .
$$

For the same set of parameters, one gets $n \gg 2 \times 10^{14} \mathrm{~cm}^{-3}$. Therefore, for both cases of weak and strong screening, there is a requirement on the minimal electron density or, equivalently, on the minimal density of the injection current in order to use the one-dimensional electrostatic screening picture for the fluctuations. Otherwise, each carrier perturbs the electrostatic potential independently and the threedimensional approach is needed. On the other hand, the assumption of the nondegenerate electron gas restricts our approach by a maximum electron concentration dependent on $T$. For temperatures in the range $10-77 \mathrm{~K}$, these maximal concentrations are estimated to be in the range $3 \times 10^{16}$ to $6 \times 10^{17} \mathrm{~cm}^{-3}$. These estimates show that the approach undertaken below covers a wide range of typical diode parameters: electron concentrations, diode lengths, and temperatures.

\section{BASIC EQUATIONS}

A semiclassical space-charge-limited transport in a ballistic conductor is completely described by the electron distribution function $F\left(X, v_{x}, t\right)$ and the electrostatic potential $\varphi(X, t)$. Here, $v_{x}$ is the $X$ component of the electron velocity and $t$ is the time. The potential $\varphi(X, t)$ inside the sample is determined by the distribution of space charge from the Poisson equation

$$
\frac{d^{2} \varphi}{d X^{2}}=\frac{q}{\kappa} N(X, t)
$$

with the boundary conditions

$$
\varphi(0, t)=\varphi_{L}, \quad \varphi(d, t)=\varphi_{R} .
$$

The voltage bias between the contacts $U=\varphi_{R}-\varphi_{L}$ is assumed to be fixed by a low-impedance external circuit. The electron density $N(X, t)$ at any plane $X$ is determined by integrating the local electron distribution function over velocities

$$
N(X, t)=\int_{-\infty}^{\infty} F\left(X, v_{x}, t\right) d v_{x}
$$

whereas the current in the external lead is given by ${ }^{9}$

$$
I(t)=-\frac{q A}{d} \int_{0}^{d}\left[\int_{-\infty}^{\infty} v_{x} F\left(X, v_{x}, t\right) d v_{x}\right] d X+C_{0} \frac{\partial U}{\partial t}
$$

where $C_{0}=\kappa A / d$ is a capacitance and $A$ the cross-sectional area. Due to a fixed-applied-voltage condition, in what follows we shall neglect the last term in Eq. (7) coming from the displacement current contribution. In addition, for simplicity, we shall omit the minus sign for the current, which is opposed to the direction of electron flow. Moreover, as will be shown below, the current is conserved along the sample due to the conservation of electron energy under ballistic motion (this is true for both the stationary current and its fluctuation). Therefore, the integration over $X$ becomes trivial and it will be disregarded.

Under ballistic motion the distribution function $F\left(X, v_{x}, t\right)$ obeys the collisionless kinetic equation

$$
\frac{\partial F}{\partial t}+v_{x} \frac{\partial F}{\partial X}+\frac{q}{m} \frac{d \varphi}{d X} \frac{\partial F}{\partial v_{x}}=0
$$


where $m$ stands for the electron effective mass. The distribution functions injected from the contacts electrons are assumed to be given as

$$
\begin{aligned}
& \left.F\left(0, v_{x}, t\right)\right|_{v_{x}>0}=F_{L}\left(v_{x}, t\right), \\
& \left.F\left(d, v_{x}, t\right)\right|_{v_{x}<0}=F_{R}\left(v_{x}, t\right) .
\end{aligned}
$$

The kinetic equation (8) with the electrostatic potential determined self-consistently from Eqs. (4) and (6) are known as the Vlasov system of equations ${ }^{42}$ describing the dynamical screening of the interaction in plasma. ${ }^{43}$

Equation (8) may also be expressed as

$$
\left.\frac{d F}{d t}\right|_{\text {trajectory }}=0,
$$

since $F$ is constant along an electron trajectory, i.e., the distribution function at any plane $X$ can be expressed through the functions $F_{k}\left(v_{x}, t\right), k=L, R$ defined at the boundaries. Each of these functions is considered to consist of two terms, a stationary part describing the stationary injection and a time-varying stochastic component. Explicitly,

$$
F_{k}\left(v_{x}, t\right)=\bar{F}_{k}\left(v_{x}\right)+\delta F_{k}\left(v_{x}, t\right), \quad k=L, R .
$$

Under nondegenerate and equilibrium conditions in the contacts, we assume for the stationary part of the injection function the half-Maxwellian distribution

$$
\bar{F}_{k}\left(v_{x}\right)=\frac{2 N_{0}}{v_{0} \sqrt{\pi}} e^{-v_{x}^{2} / v_{0}^{2}}
$$

with $v_{x}>0$ for $k=L$ and $v_{x}<0$ for $k=R$. Here, $N_{0}$ is the density of electrons injected from the contacts and $v_{0}$ $=\sqrt{2 k_{B} T / m}$ is the thermal velocity. The contact distribution functions (12) are normalized in such a way that the integration over a half-velocity space yields the density of electrons injected from the contact

$$
N_{0}=\int_{v_{x}>0} \bar{F}_{L}\left(v_{x}\right) d v_{x}=\int_{v_{x}<0} \bar{F}_{R}\left(v_{x}\right) d v_{x} .
$$

The stochastic terms $\delta F_{k}, k=L, R$ in Eq. (11) are the only sources of noise under ballistic transport considered here, since the electron motion between the contacts is noiseless. Their equal-time correlation, due to equilibrium conditions, is given by ${ }^{44}$

$$
\begin{aligned}
\left\langle\delta F_{k}\left(v_{x}, t\right) \delta F_{k^{\prime}}\left(v_{x}^{\prime}, t\right)\right\rangle= & C \bar{F}\left(v_{x}\right)\left[1-\bar{F}\left(v_{x}\right)\right] \\
& \times \delta_{k k^{\prime}} \delta\left(v_{x}-v_{x}^{\prime}\right),
\end{aligned}
$$

where the constant $C$ is determined from the normalization condition. Since the injected electron gas is nondegenerate, $\bar{F} \ll 1$, and the factor $1-\bar{F}$ will be ignored.

As a consequence of the fluctuations inside the contacts (whose origin is ultimately the carrier scattering processes), both the electron distribution function and electrostatic potential in the ballistic sample fluctuate, leading to the current fluctuations. These quantities will be presented as a sum of stationary and fluctuating contributions: $F\left(X, v_{x}, t\right)$
$=\bar{F}\left(X, v_{x}\right)+\delta F\left(X, v_{x}, t\right), \quad N(X, t)=\bar{N}(X)+\delta N(X, t), \quad \varphi(X, t)$ $=\bar{\varphi}(X)+\delta \varphi(X, t)$, and $I(t)=\bar{I}+\delta I(t)$.

Introducing the Fourier transform for the fluctuations of the distribution function $\delta F_{\omega}\left(X, v_{x}\right)$ and the potential $\delta \varphi_{\omega}(X)$, the kinetic equation takes on the form

$$
-i \omega \delta F_{\omega}+v_{x} \frac{\partial \delta F_{\omega}}{\partial X}+\frac{q}{m} \frac{d \bar{\varphi}}{d X} \frac{\partial \delta F_{\omega}}{\partial v_{x}}+\frac{q}{m} \frac{\partial \bar{F}}{\partial v_{x}} \frac{d \delta \varphi_{\omega}}{d X}=0,
$$

with the boundary conditions at the contacts

$$
\begin{aligned}
& \left.\delta F_{\omega}\left(0, v_{x}\right)\right|_{v_{x}>0}=\delta F_{L}^{\omega}\left(v_{x}\right), \\
& \left.\delta F_{\omega}\left(L, v_{x}\right)\right|_{v_{x}<0}=\delta F_{R}^{\omega}\left(v_{x}\right),
\end{aligned}
$$

where $\delta F_{L}^{\omega}$ and $\delta F_{R}^{\omega}$ are the Fourier transforms of the stochastic functions from Eq. (11). The equation for the fluctuating potential $\delta \varphi_{\omega}$ is trivially obtained from Eqs. (4) and (6),

$$
\frac{d^{2} \delta \varphi_{\omega}}{d X^{2}}=\frac{q}{\epsilon} \int \delta F_{\omega}\left(X, v_{x}\right) d v_{x}
$$

the boundary conditions for which follows from Eq. (5),

$$
\delta \varphi_{L}^{\omega}(0)=0, \quad \delta \varphi_{R}^{\omega}(d)=0 .
$$

Below we restrict ourselves to the calculation of the lowfrequency plateau of the noise spectrum; thus one can omit the term proportional to $\omega$ in Eq. (15). It can be shown that this approximation is valid if the shortest fluctuation period in $\delta F_{k}(t)$ is considered to be sufficiently greater than the average electron transit time $\tau_{T}$ across the diode, i.e., $\omega$ $\ll \tau_{T}^{-1}$. Thus, the above self-consistent equations completely describe the stationary transport and low-frequency fluctuations in the ballistic sample, and below we shall omit the index $\omega$.

It is advantageous to rescale all the variables as follows:

$$
\begin{gathered}
w=\frac{v_{x}}{v_{0}}, \quad x=\frac{X}{L_{D}}, \quad \psi=\frac{q \bar{\varphi}}{k_{B} T} \\
n=\frac{\bar{N}}{2 N_{0}}, \quad f=\bar{F} \frac{v_{0}}{2 N_{0}}, \quad \delta f=\delta F \frac{v_{0}}{2 N_{0}} .
\end{gathered}
$$

In such units the basic equations contain only two dimensionless parameters: (i) the length of the sample (or the screening parameter) $\lambda=d / L_{D}^{0}$, where $L_{D}^{0}$ $=\sqrt{\epsilon k_{B} T /\left(2 q^{2} N_{0}\right)}$ is the Debye screening length corresponding to the electron density $2 N_{0}$, and (ii) the applied voltage bias $V=q U /\left(k_{B} T\right)$. Below we use the dimensionless variables in all the equations.

\section{STEADY-STATE PROBLEM}

The calculation of fluctuations in the ballistic conductor requires the knowledge of the stationary distribution of electrostatic field, which, in turn, can be determined by solving the full steady-state problem. The self-consistent steady-state problem can be solved as follows. First, we solve the station- 
ary collisionless kinetic equation for the distribution function $f(x, w)$

$$
w \frac{\partial f}{\partial x}+\frac{1}{2} \frac{d \psi}{d x} \frac{\partial f}{\partial w}=0
$$

at a given electrostatic potential $\psi(x)$. Integrating $f(x, w)$ over $w$, we then find the electron density profile $n(\psi)$ in terms of the potential $\psi(x)$. Then we should solve the Poisson equation

$$
\frac{d^{2} \psi}{d x^{2}}=n(\psi),
$$

with the boundary conditions

$$
\psi(0) \equiv \psi_{L}=0, \quad \psi(\lambda) \equiv \psi_{R}=V .
$$

Here, we set the zero value of the potential at the left contact.

\section{A. Stationary distribution function}

To solve the stationary kinetic equation (20), we have to specify the boundary conditions for this equation at a given $\psi(x)$. Generally, the nonstationary kinetic equation (8) and the distribution functions (9) of injected electrons completely determine the nonstationary solution $f(x, w, t)$. However, the steady-state equation (20) requires a specification of the boundary conditions for the distribution function of all the electrons: those injected from the contacts into the sample and those leaving the sample. Let the space charge in the sample be such that a potential minimum $\psi_{m}$ occurs at $x$ $=x_{m}$, which acts as a potential barrier for electrons. We define the total electron energy $\varepsilon_{t}=w^{2}-\psi(x)$. For a given potential, the distribution function should consist of the terms originating from two electron streams injected by the left and right contacts. Electrons injected from each of the contacts fall into two groups depending on their injecting energies. If the initial energy is higher than the height of the barrier, electrons obviously reach the opposite contact and contribute to the electric current. These electrons are not reflected back. Note that the height of the barrier is different for the electrons injected from the left and right contacts. For those injected from the left, it is $\psi_{L}-\psi_{m}=V_{m}$, which is the potential minimum depth, while for those injected from the right, it is $\psi_{R}-\psi_{m}=V+V_{m}$ (see Fig. 1). Accordingly, the lower bounds for the velocities of the transmitted electrons are given by

$$
\begin{gathered}
w_{L}=\sqrt{\psi_{L}-\psi_{m}}=\sqrt{V_{m}}, \\
w_{R}=\sqrt{\psi_{R}-\psi_{m}}=\sqrt{V_{m}+V} .
\end{gathered}
$$

Electrons of the second group, which we shall call the reflected electrons, are reflected by the barrier and do not contribute to the current (however, both groups affect the electrostatic potential). An electron from the second group being injected with a velocity $w$ returns to the contact with the opposite velocity of the same value $-w$. Taking into account the above consideration, the electron distribution function $f(x, w)$ at any plane $x$ may be written as

$$
f=f_{L, t}+f_{L, r}+f_{R, t}+f_{R, r},
$$

where the indices $L$ and $R$ refer to the left and right contacts, and the indices $t$ and $r$ distinguish the transmitted and reflected groups of carriers, respectively. The boundary conditions for these functions read

$$
\begin{gathered}
f_{L, t}\left(0, w_{c}\right)=f_{L}\left(w_{c}\right) \theta\left(w_{c}-w_{L}\right), \\
f_{L, r}\left(0, w_{c}\right)=f_{L}\left(w_{c}\right) \theta\left(w_{L}^{2}-w_{c}^{2}\right), \\
f_{R, t}\left(\lambda, w_{c}\right)=f_{R}\left(w_{c}\right) \theta\left(-w_{c}-w_{R}\right), \\
f_{R, r}\left(\lambda, w_{c}\right)=f_{R}\left(w_{c}\right) \theta\left(w_{R}^{2}-w_{c}^{2}\right),
\end{gathered}
$$

where $w_{c}$ is the $x$ velocity component of injected electrons at the contacts, $\theta$ is the Heaviside step function, and the distribution function of injected electrons is determined by Eq. (12), which in dimensionless units reads

$$
f_{L}\left(w_{c}\right)=f_{R}\left(w_{c}\right)=\frac{1}{\sqrt{\pi}} e^{-w_{c}^{2}} .
$$

We can solve now the collisionless kinetic equation (20) explicitly for a given potential profile $\psi(x)$. Indeed, one can easily see that its solution is an arbitrary function dependent on the total electron energy $\mathcal{F}\left(\varepsilon_{t}\right)=\mathcal{F}\left(w^{2}-\psi(x)\right)$. The boundary conditions (25) determine the shape of this function. By using the electron-energy conservation law

$$
w^{2}-\psi(x)=w_{c}^{2}-\psi_{k}, \quad k=L, R,
$$

where $w_{c}$ and $\psi_{k}$ are the parameters at the contacts, we exclude $w_{c}$ in the boundary conditions (25) and obtain the contributions in the distribution function as

$$
\begin{gathered}
f_{L, t}(x, w)=\frac{1}{\sqrt{\pi}} \theta\left(w-w_{*}(x)\right) e^{-w^{2}+\psi(x)-\psi_{L},} \\
f_{R, t}(x, w)=\frac{1}{\sqrt{\pi}} \theta\left(-w-w_{*}(x)\right) e^{-w^{2}+\psi(x)-\psi_{R},} \\
f_{k, r}(x, w)=\frac{1}{\sqrt{\pi}} \theta\left(w_{*}^{2}(x)-w^{2}\right) e^{-w^{2}+\psi(x)-\psi_{k},}
\end{gathered}
$$

where $k=L, R$, and the functions $f_{L, t}$ and $f_{R, t}$ for the transmitted electrons are defined in the whole range $0<x<\lambda$, whereas the expressions for the reflected electrons $f_{L, r}$ and $f_{R, r}$ are valid in the intervals $0<x<x_{m}$ and $x_{m}<x<\lambda$, respectively. In Eqs. (28) we have introduced the quantity

$$
w_{*}(x)=\sqrt{\psi(x)-\psi_{m}},
$$

which has a meaning of the maximal velocity of reflected electrons at a point $x$. For the sake of clarity, in Fig. 2 we show the electron trajectories in the phase space $(x, w)$ corresponding to different electron groups. It is worth stressing that the distributions (28) depend on the local potential $\psi(x)$ and the potential minimum $\psi_{m}$ as well, i.e., the distribution function depends nonlocally on the potential profile.

Summing up all the contributions (28), the total distribution function takes on the form 


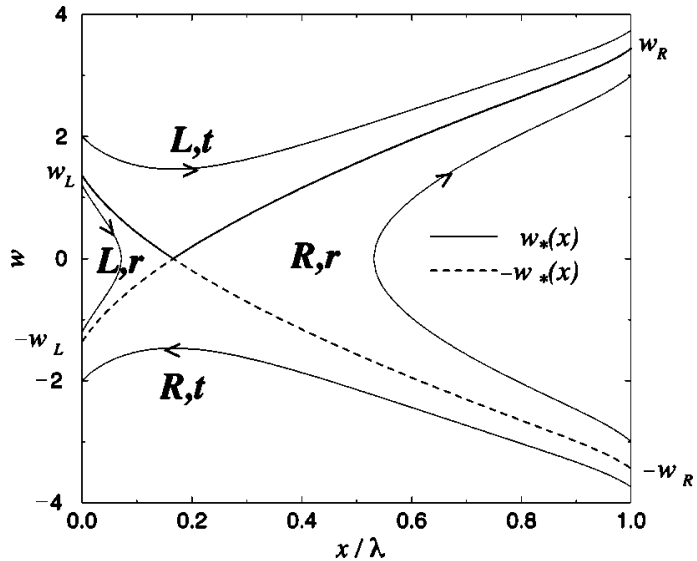

FIG. 2. Typical electron trajectories in the phase space $(x, w)$ for different electron groups: $L$ and $R$ refer to the carriers originated from the left and right contacts, and $t$ and $r$ refer to the transmitted and reflected groups of carriers. The separating curves are the critical velocities $\pm w_{*}(x)$, which intersect at the point of the potential minimum $\left(x_{m}, 0\right)$. The results are for $\lambda=30, V=10$.

$$
f(x, w)=\frac{1}{\sqrt{\pi}} e^{-w^{2}+\psi(x)} \times \begin{cases}e^{-\psi_{L}}, & w \geqslant \mp w_{*}(x) \\ e^{-\psi_{R}}, & w<\mp w_{*}(x) .\end{cases}
$$

Here, and throughout the paper, we shall use the upper sign for the left side of the potential minimum $0<x<x_{m}$ and the lower sign for the right side of the potential minimum $x_{m}$ $<x<\lambda$. It is seen that the obtained distribution function is discontinuous on $w$ at the points where $w=w_{*}(x)$ (see also Fig. 7 discussed below). It is not surprising, since only a discontinuous solution can satisfy the first-order equation (20) and simultaneously two different arbitrary functions given at the boundaries.

\section{B. Electron density}

The distribution function (30) allows us to find the electron density at a slice $x$ as

$$
\begin{aligned}
n(x)= & \frac{1}{\sqrt{\pi}} e^{\psi(x)}\left[e^{-\psi_{L}} \int_{\mp w_{*}(x)}^{\infty} e^{-w^{2}} d w\right. \\
& \left.+e^{-\psi_{R}} \int_{-\infty}^{\mp w_{*}(x)} e^{-w^{2}} d w\right] \\
= & \frac{1}{2} e^{\psi(x)}\left(e^{-\psi_{L}\left\{1 \pm \operatorname{erf}\left[w_{*}(x)\right]\right\}}\right. \\
& +e^{\left.-\psi_{R}\left\{1 \mp \operatorname{erf}\left[w_{*}(x)\right]\right\}\right),}
\end{aligned}
$$

where $\operatorname{erf}(x)=(2 / \sqrt{\pi}) \int_{0}^{x} e^{-u^{2}} d u$ stands for the error function. By using the values for the potential (22) at the contacts and denoting

$$
\beta_{1}=1+e^{-V}, \quad \beta_{2}=1-e^{-V},
$$

the electron density can be written as a function of $\psi$,

$$
n(\psi)=\frac{1}{2} e^{\psi}\left[\beta_{1} \pm \beta_{2} \operatorname{erf}\left(\sqrt{\psi-\psi_{m}}\right)\right]
$$

where, as before, the upper sign applies in the interval 0 $<x<x_{m}$ and the lower sign applies in the interval $x_{m}<x$ $<\lambda$. Note that in equilibrium, $V=0, \beta_{1}=2, \beta_{2}=0$, the Boltzmann distribution $n(x)=e^{\psi(x)}$ is recovered throughout the sample. Furthermore, Eq. (33) is valid for a singleinjection (vacuum) diode, assuming $\beta_{1}=\beta_{2}=1.8,9$

In the following we shall use the shifted potential measured from the minimum

$$
\eta(x)=\psi(x)-\psi_{m},
$$

and Eq. (33) in terms of the new variable $\eta$ becomes

$$
n(\eta)=n_{m} e^{\eta}[1 \pm \beta \operatorname{erf} \sqrt{\eta}],
$$

where $n_{m}=\frac{1}{2} \beta_{1} e^{-V_{m}}$ is the electron density at the potential minimum, and

$$
\beta \equiv \frac{\beta_{2}}{\beta_{1}}=\tanh \left(\frac{V}{2}\right)
$$

\section{Steady-state electrostatic potential}

Having found the analytical expression for $n(\psi)$, we have to use it to solve the Poisson equation (21). Multiplying both sides of Eq. (21) by $d \psi / d x$ and integrating, one gets

$$
\left(\frac{d \psi}{d x}\right)^{2}=2 \int_{\psi_{m}}^{\psi} n(\widetilde{\psi}) d \widetilde{\psi}
$$

where we have used the property of the potential minimum $\left.(d \psi / d x)\right|_{x=x_{m}}=0$. Changing to the shifted-potential variable $\eta$ and carrying out the integration, one gets

$$
l_{m}^{2}\left(\frac{d \eta}{d x}\right)^{2}=h_{V}^{\mp}(\eta)
$$

where $1 / l_{m}^{2}=2 n_{m}=\beta_{1} e^{-V_{m}}$ and the function

$$
h_{V}^{\mp}(\eta)=e^{\eta}-1 \pm \beta\left(e^{\eta} \operatorname{erf} \sqrt{\eta}-\frac{2}{\sqrt{\pi}} \sqrt{\eta}\right),
$$

depends on the applied voltage $V$ through $\beta$. Taking into account $d \eta / d x<0$ for $0<x<x_{m}$ and $d \eta / d x>0$ for $x_{m}<x$ $<\lambda$, the electric field is given by

$$
E=-\frac{d \eta}{d x}=\left\{\begin{array}{lc}
\sqrt{h_{V}^{-}(\eta)} / l_{m}, & 0<x<x_{m} \\
-\sqrt{h_{V}^{+}(\eta)} / l_{m}, & x_{m}<x<\lambda
\end{array}\right.
$$

which is measured in units of $k_{B} T / q L_{D}^{0}$. Integrating Eq. (38), one obtains the distribution of the potential in an implicit form,

$$
x=\left\{\begin{array}{l}
l_{m} \int_{\eta}^{\eta_{L}} \frac{d \eta}{\sqrt{h_{V}^{-}(\eta)}}, \quad 0<x<x_{m} \\
\lambda-l_{m} \int_{\eta}^{\eta_{R}} \frac{d \eta}{\sqrt{h_{V}^{+}(\eta)}}, \quad x_{m}<x<\lambda
\end{array}\right.
$$

where the boundary conditions for $\eta(x)$ are

$$
\eta(0) \equiv \eta_{L}=V_{m}, \quad \eta(\lambda) \equiv \eta_{R}=V_{m}+V .
$$


For the given $V, \lambda$, the only unknown parameter in Eqs. (41) is the potential minimum $V_{m}$. The latter is found by matching Eq. (41) at $x=x_{m}$, where $\eta\left(x_{m}\right)=0$, and one gets

$$
\lambda_{m}(V)=\int_{0}^{V_{m}(V)} \frac{d \eta}{\sqrt{h_{V}^{-}(\eta)}}+\int_{0}^{V_{m}(V)+V} \frac{d \eta}{\sqrt{h_{V}^{+}(\eta)}},
$$

where

$$
\lambda_{m}=\lambda \sqrt{2 n_{m}}
$$

is the screening parameter renormalized to the electron density at the potential minimum rather than to the contact electron density as before.

\section{Steady-state current}

This brief description of the steady state is then completed by the expression for the stationary current. Substituting the distribution function into Eq. (7) and changing the variables with the help of Eq. (27) as $w d w=w_{c} d w_{c}$, one obtains

$$
\begin{aligned}
\bar{I} & =2 \sqrt{\pi} I_{c}\left[\int_{w^{*}(x)}^{\infty} f_{L, t}(x, w) w d w+\int_{-\infty}^{-w^{*}(x)} f_{R, t}(x, w) w d w\right] \\
& =2 \sqrt{\pi} I_{c}\left[\int_{w_{L}}^{\infty} f_{L, t}\left(0, w_{c}\right) w_{c} d w_{c}-\int_{w_{R}}^{\infty} f_{R, t}\left(\lambda, w_{c}\right) w_{c} d w_{c}\right],
\end{aligned}
$$

where

$$
I_{c}=\frac{1}{\sqrt{\pi}} q N_{0} v_{0} A=q N_{0} \bar{v} A
$$

is the emission current from each contact (limiting value for the total current at $\left.V \rightarrow \infty, V_{m} \rightarrow 0\right)$ and $\bar{v}=v_{0} / \sqrt{\pi}$ $=\sqrt{2 k_{B} T /(\pi m)}$ is the average velocity of the injected electrons with the half-Maxwellian distribution. Only the part of the distribution function corresponding to the transmitted electrons has been taken into account, since the reflected carriers gives no contribution to the current. [This is in contrast to the case of the calculation of the electron density (31) for which both transmitted and reflected carriers contribute.] It is seen from Eq. (45) that the current is the same for any section $x$ of the sample, given by its value at the injected contacts. Substituting the functions (25) into Eq. (45) and carrying out the integration, we obtain the current as a sum of two opposing currents: $I_{L R}$ and $I_{R L}$ caused by the injection from the left and right contacts, respectively,

$$
\bar{I}=I_{c} e^{-V_{m}}-I_{c} e^{-V_{m}-V} \equiv I_{L R}-I_{R L} .
$$

The formula for the current may be written through the electron density at the potential minimum, that is,

$$
\bar{I}=2 n_{m} I_{c} \beta=q N_{m} \bar{v} A \tanh \left(\frac{q U}{2 k_{B} T}\right),
$$

where $N_{m}=2 N_{0} n_{m}$. This formula justifies the usage of the term "virtual cathode" referred to the location of the potential minimum, since it is seen that the current is determined by the injection of the electron density $N_{m}$ from the virtual cathode. The additional $\tanh ()$ factor takes into account the

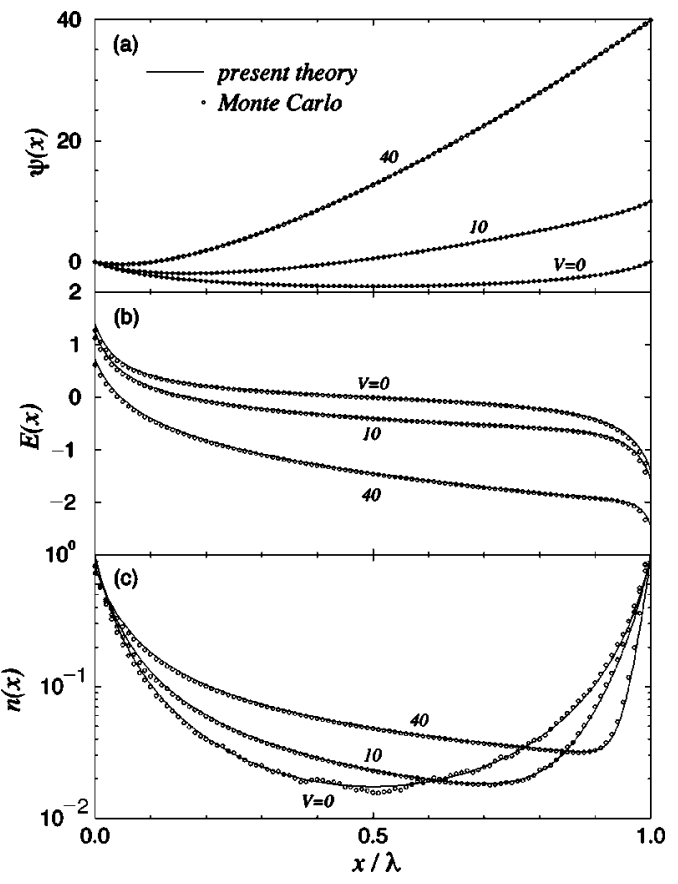

FIG. 3. Spatial profiles for the normalized quantities: (a) potential $\psi$, (b) electric field $E$, and (c) electron density $n$ (all solid lines) for $\lambda=30.9$ and several applied biases $V$. The corresponding units are $k_{B} T / q, k_{B} T / q L_{D}^{0}$, and $2 N_{0}$. The results are shown to be in excellent agreement with the Monte Carlo simulations (Ref. 48) (symbols).

injection in the opposite direction, and it tends to 1 at $q U$ $\gg k_{B} T$. (For the vacuum diode case, this factor is set to 1 because of only one injecting contact.)

Summarizing this section, we note that the above relations solve completely the steady-state problem for the ballistic two-terminal conductor: Eqs. (41) determine the distribution of the potential across the diode in an implicit form, and Eqs. (43) and (47) determine the current-voltage characteristics. Note that in Eq. (47) the current depends on voltage through both the explicit term $e^{-V}$ and the potential minimum $V_{m}$, which is a function of voltage. Equations (33), (39)-(43), and (47) may be viewed as an extension of the Fry-Langmuir theory for a single-injection vacuum diode ${ }^{45-47}$ to the double-injection case. The Fry-Langmuir formulas are obtained by setting $\beta_{1}=\beta_{2}=1, I_{R L}=0$.

\section{E. Results}

Figure 3 shows the typical spatial distributions of the potential $\psi$, electric field $E$, and electron density $n$ along the diode obtained from Eqs. (35), (40), (41), and (43). With the aim to compare our theory with the results of the Monte Carlo simulations, ${ }^{48}$ we present the spatial profiles for the value of $\lambda=30.9$ and various applied biases $V$. As it is seen from the figure, the agreement is excellent for all the quantities.

The space-charge-limited conduction is characterized by a strong transport inhomogeneity in the ballistic region and by the presence of the potential minimum [Fig. 3(a)] due to the injected space charge. The minimum acts as a barrier for the electrons moving in both directions. Its magnitude progres- 


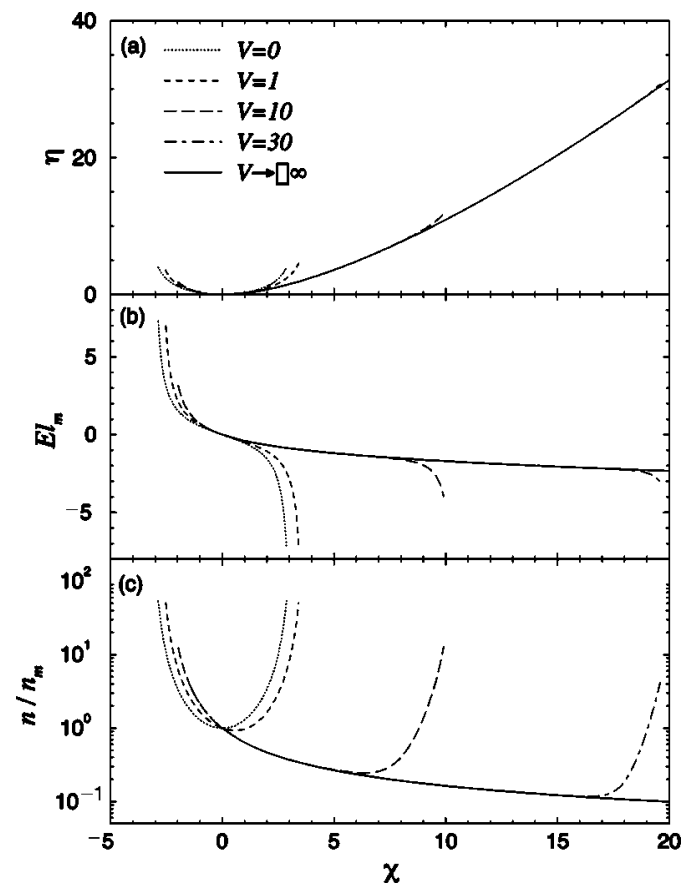

FIG. 4. Profiles for the steady-state quantities in units of the potential-minimum parameters: (a) potential, (b) electric field, and (c) electron density. The limiting universal profiles at $V \rightarrow \infty$ are shown by solid lines.

sively decreases as the applied bias is increased, simultaneously shifting towards the left contact on which the potential is fixed.

The obtained solutions are determined by two dimensionless parameters: $\lambda$ and $V$. The spatial distributions, however, may be presented in a more universal form by using for scaling the potential minimum parameters. We define the new coordinate $\chi=\left(x-x_{m}\right) / l_{m}$, where the characteristic length $l_{m}=\left(2 n_{m}\right)^{-1 / 2}$, dependent on the electron density at the potential minimum, has been introduced in Sec. IV C. This is equivalent to scale the original coordinate $X$ in units of the screening length referred to the electron density at the potential minimum rather than to the contact electron density. In such a unit, the parameter $\lambda$ is scaled away from the equation for the potential, remaining only in the upper and lower bounds of the function variation. Explicitly, Eq. (41) becomes

$$
\chi= \begin{cases}-\int_{0}^{\eta}\left[d \eta / \sqrt{h_{V}^{-}(\eta)}\right], & -x_{m} / l_{m}<\chi<0, \\ \int_{0}^{\eta}\left[d \eta / \sqrt{h_{V}^{+}(\eta)}\right], & 0<\chi<\left(\lambda-x_{m}\right) / l_{m} .\end{cases}
$$

Therefore, all the solutions may be presented as a oneparameter family of curves dependent on the applied bias $V$ only. Moreover, at high-voltage limit $V \geqq 5, \beta \rightarrow 1$, the functions $h_{V}^{ \pm}(\eta)$ become independent of bias, and the spatial distributions tend to the limiting universal profiles for each quantity which are free from any parameter. This is valid for all the spatial characteristics as it is seen from Fig. 4, where the potential $\eta=\psi-\psi_{m}$, the electric field $E l_{m}$, and the electron density $n / n_{m}$ are plotted. Moreover, at this limit the part of each profile at $\chi<0$ tends to vanish (the potential mini-

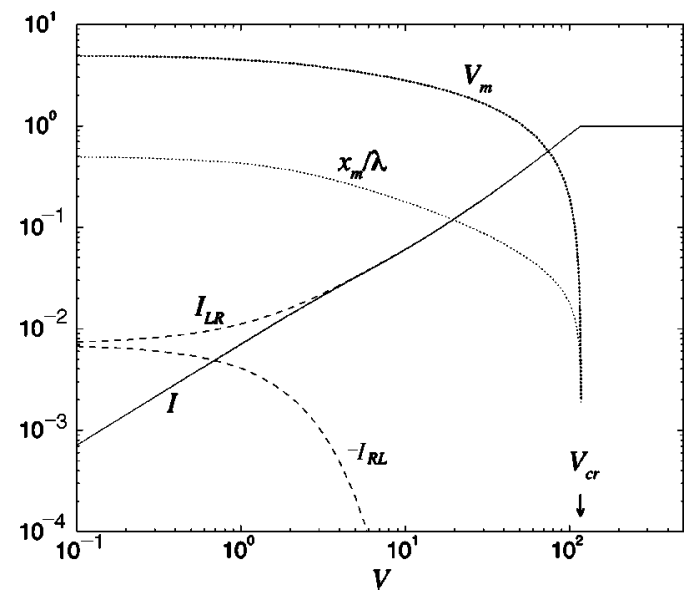

FIG. 5. Current and its components coming from two opposite electron flows $\bar{I}=I_{L R}-I_{R L}$ (in units of $I_{c}$ ) vs bias $V$ for $\lambda=50$. The height of the potential barrier $V_{m}$, and its location $x_{m} / \lambda$ are also shown.

mum approaches the left contact), which leads to the validity of the virtual-cathode approximation with the boundary condition $E(0)=0$. The universal profiles obey the asymptotic behavior at $\chi \rightarrow \infty: \quad \eta(\chi)=\frac{3}{4} a \chi^{4 / 3}, E l_{m}=-a \chi^{1 / 3}, n / n_{m}$ $=\frac{2}{3} a \chi^{-2 / 3}$, where $a=(3 / \pi)^{1 / 3} \approx 0.9847$. Going back from $\chi$ to the $x$ coordinate, and using the Child law (54), which will be discussed below, one can obtain the asymptotic formula for the potential profile $\eta(x)=V(x / \lambda)^{4 / 3}, x \rightarrow \infty$. The latter is valid not only for the present nondegenerate-electron-gas model, but for an arbitrary distribution function of the injected electrons, provided $V \rightarrow \infty, \lambda \rightarrow \infty$ (virtual-cathode approximation). ${ }^{49}$ The related formulas for $E(x), n(x)$ may also be obtained by taking the derivatives.

The choice of the potential minimum parameters as reference coordinates is of traditional use in vacuum-diode literature. ${ }^{8,9,46}$ Since only one contact (cathode) is considered as injected for these diodes, $\beta=1$ for any bias, and the universal potential profile independent of the diode parameters is obtained for any bias, as it was tabulated in the original work by Langmuir. ${ }^{46}$ For the case of the two-terminal semiconductor diode, that universality is broken at low and moderate biases due to the contribution to the current from the second injecting contact, but it is recovered however at high biases $V \rightarrow \infty$ when the influence of the second contact becomes negligible, as it is demonstrated in Fig. 4. We remark additionally that the virtual-cathode approximation is only valid when besides $V \rightarrow \infty$ another condition is fulfilled simultaneously, $V<V_{c r}$. Otherwise, the transport is no longer limited by the space charge, the current saturates at $\bar{I}=I_{c}$, and the value of the electric field at the left injecting contact is no longer zero, $E(0)<0$. This change in the transport regime is clearly seen in Fig. 5, where the current and its components coming from two opposite electron flows $\bar{I}$ $=I_{L R}-I_{R L}$ versus bias $V$ are plotted for a particular value of $\lambda$. It is seen that the current is an increasing function of the bias up to the critical value $V_{c r}$, after which it is saturated at $\bar{I}=I_{c}$. At that point the potential minimum vanishes. For $V$ $\$ 5$ the contribution to the current from the right-contact electrons is also essential.

The $I-V$ curves for different levels of screening are shown 


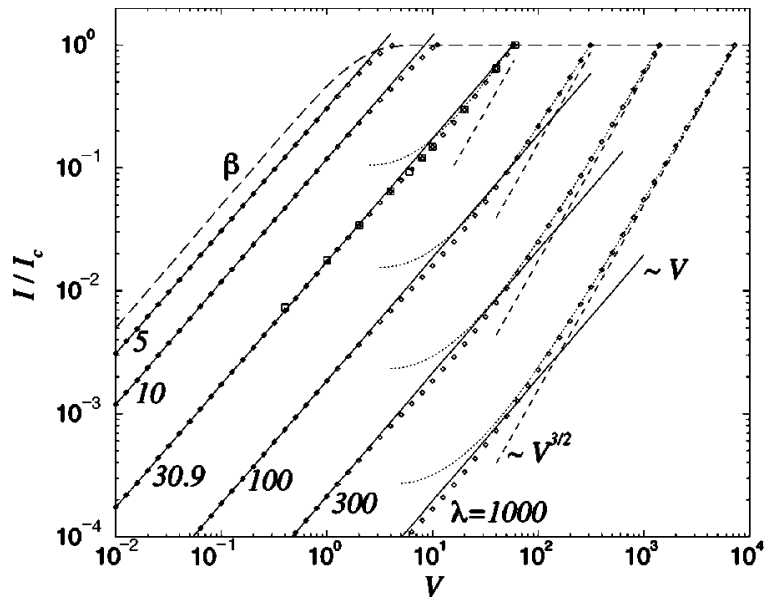

FIG. 6. Current-voltage characteristics for different levels of screening $\lambda$ obtained as solutions of the present theory (diamonds). For comparison, the approximate solutions are shown: linear dependences given by Eq. (50) (solid); Langmuir formula given by Eq. (54) (dots); the Child 3/2-power law given by Eq. (53) (dashes); parameter $\beta$, shown by long dashes, when approaching to 1 indicates the bias $(V \approx 5)$ over which the effect of the second contact on $I-V$ curves becomes negligible. Monte Carlo simulation results (Ref. 48) for $\lambda=30.9$ are shown by squares.

in Fig. 6. We have checked that the obtained solutions are in excellent agreement with the Monte Carlo simulations ${ }^{48}$ (the case of $\lambda=30.9$ is compared in the figure).

The analysis shows the following behavior. At low biases, the $I-V$ curves are linear for all $\lambda$ despite the fact that the transport is space-charge-limited. The curves for this case are described by

$$
I_{\text {lin }} \approx I_{c} V e^{-V_{m}^{0}}, \quad V \lesssim 1,
$$

where $V_{m}^{0}$ is the equilibrium value of the potential minimum whose value depends on $\lambda$. In the range $1 \leqq V \leqq 10$ the $I-V$ curves deviate to sublinear dependence. At high biases, starting approximately at $V \approx 5$ where $\beta \rightarrow 1$, the effect of injection from the second contact becomes negligible, $I_{R L} \ll I_{L R}$ (see also Fig. 5). Furthermore, for $V \gg V_{m} \gg 1$ the analytical solution may be found. In this regime, the function $h^{+}(\eta)$ may be approximated by leading-order terms of a series expansion in a similar way as in the case of a vacuum diode ${ }^{47}$

$$
h^{+}(\eta) \approx 2 \sqrt{\eta / \pi}-1, \quad \eta \rightarrow \infty .
$$

In this regime from Eq. (41b) one can write

$$
\lambda-x_{m} \approx l_{m} \int_{\pi / 4}^{V+V_{m}} \frac{d \eta}{(2 \sqrt{\eta / \pi}-1)^{1 / 2}},
$$

from which by using $l_{m} \approx 1 / \sqrt{J}$ follows the Langmuir formula ${ }^{9,46,47}$

$$
I_{\text {Lang }}=\frac{8}{9} \sqrt{\pi} I_{c} \frac{\left(V+V_{m}\right)^{3 / 2}}{\left(\lambda-x_{m}\right)^{2}}\left[1+\frac{3}{\sqrt{(4 / \pi)\left(V+V_{m}\right)}}\right] .
$$

In Fig. 6 we present the curves calculated from this formula, and they are seen to describe accurately the $I-V$ characteristics for the highest biases. For higher $\lambda$, the range of biases where this formula may apply is wider. In the asymptotic

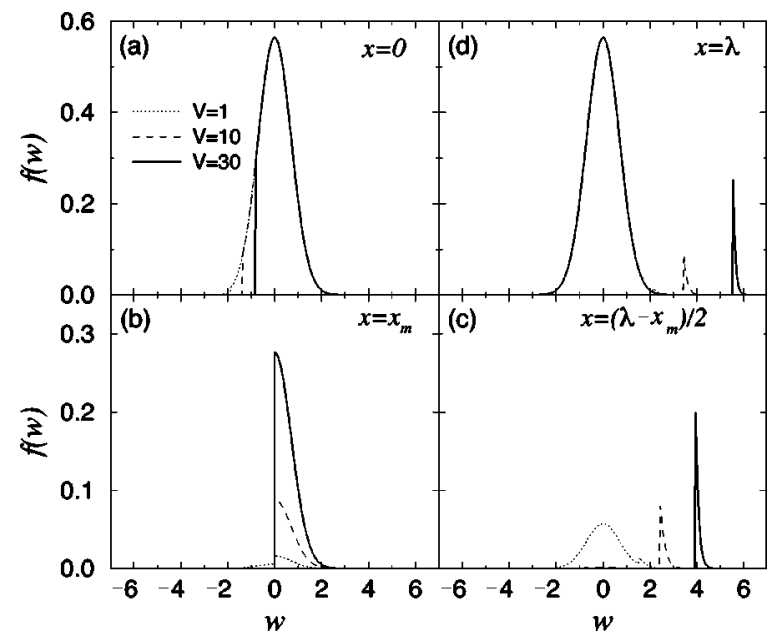

FIG. 7. Electron distribution function over dimensionless velocities $f(w)$ at different sections $x$ of the diode for several biases $V$ and $\lambda=30$.

limit $V \rightarrow \infty, \lambda \rightarrow \infty$, one may neglect $x_{m}$ and $V_{m}$ as compared to $\lambda$ and $V$, respectively, and one obtains the Child 3/2-power law, which is free from the potential minimum parameters

$$
I_{\text {Child }}=\frac{8}{9} \sqrt{\pi} I_{c} \frac{V^{3 / 2}}{\lambda^{2}}
$$

It is seen from the figure, that this asymptotic formula accurately describes the $I-V$ curves only at very high values of the parameters: $\lambda \geq 10^{3}, \quad V \geqq 10^{3}$. However, as we have discussed earlier, there is a relevant difference between the semiconductor and vacuum ballistic diodes. In vacuum diodes the applied voltage may be quite large without breaking down the ballistic transport regime. In contrast, in solids, electrons even for a pure material interact with a lattice. Under a low-bias regime this interaction is weak, but it becomes quite strong at high biases due to the significant increase of the electron energy. For instance, the threshold for the optical phonon generation in GaAs is about $0.036 \mathrm{eV}$, which corresponds to $V \approx 40$ at $T \sim 10 \mathrm{~K}$. Thus, one cannot bias the sample to the voltage more than that value, since a strong interaction with the lattice will break down the ballistic regime. The allowed range of biases is typically restricted by $U \leq 50 k_{B} T / q$. Then, for real structures the ballistic lengths $\lambda$ is well below 100. Therefore, the Child 3/2-power law is hard to achieve in semiconductor ballistic $n-i-n$ diodes, and one should use the full set of formulas described in the present paper from which follows the linear or sublinear $I-V$ dependences in a wide range of biases even under a strong limitation of transport by a space charge.

Finally, Fig. 7 illustrates the stationary electron distribution function over velocities $f(w)$ at different sections of the diode for several biases $V$. The distribution functions are discontinuous at $w=w^{*}(x) \operatorname{sgn}\left(x-x_{m}\right)$, as discussed in Sec. IV A. It is interesting to note that at high biases the arriving electrons at the right (receiving) contact exhibit a sharp peak separated in energy from the intrinsic contact electrons [Fig. $7(d)]$ and thus may be distinguished in an experiment. ${ }^{50,51}$ While the injected carriers are uncorrelated, electrons arriv- 
ing at the receiving contact that belong to that peak exhibit correlations in energy. This interesting result will be discussed in Sec. V H.

\section{FLUCTUATION PROBLEM}

We will find here the fluctuations of the distribution function, electron density, electrostatic potential, and current in the ballistic region of the diode, which are caused by the fluctuations in the contacts. To solve the fluctuation problem (15)-(18) self-consistently, we undertake the same approach as used above for the steady-state problem. First, we calculate the fluctuation of the distribution function $\delta f$ in a given electrostatic potential $\psi(x)+\delta \psi(x)$ by solving the perturbed kinetic equation

$$
w \frac{\partial \delta f}{\partial x}+\frac{1}{2} \frac{d \psi}{d x} \frac{\partial \delta f}{\partial w}+\frac{1}{2} \frac{\partial f}{\partial w} \frac{d \delta \psi}{d x}=0
$$

The solutions for $\delta f(x, w)$ for different groups of electrons are derived in the Appendix, where we also present the corresponding electron density fluctuations $\delta n(x)$ obtained by integration over velocities. The fluctuations $\delta n$, which are the functions of $\delta \psi$ and the contact fluctuations $\delta f_{L}$ and $\delta f_{R}$, should then be substituted into the perturbed Poisson equation

$$
\frac{d^{2} \delta \psi}{d x^{2}}=\delta n(x)
$$

with the boundary conditions

$$
\delta \psi(0)=\delta \psi(\lambda)=0
$$

to find the self-consistent fluctuations of the potential $\delta \psi$.

The fluctuations of the distribution functions of injected electrons $\delta f_{L}$ and $\delta f_{R}$ are supposed to be given by the correlator (14). It is advantageous, however, to express them through the injected current fluctuations. For each injection energy $\varepsilon \equiv w_{c}^{2}$, their relationship is given by

$$
\delta I_{k}(\varepsilon)=\sqrt{\pi} I_{c} \delta f_{k}(\varepsilon), \quad k=L, R,
$$

where $\delta I_{k}$ is the low-frequency Fourier component of the injection current fluctuation, and $I_{c}$ is the mean emission current defined by Eq. (46). The correlator for $\delta I_{k}$ is obtained from that for $\delta F_{k}$ given by Eq. (14), and one gets

$$
\left\langle\delta I_{k}(\varepsilon) \delta I_{k^{\prime}}\left(\varepsilon^{\prime}\right)\right\rangle=2 q I_{c} \Delta f e^{-\varepsilon} \delta_{k k^{\prime}} \delta\left(\varepsilon-\varepsilon^{\prime}\right),
$$

with $\Delta f$ the frequency bandwidth. The obtained correlator shows that the electrons with different energies are uncorrelated, which is a consequence of the Poissonian injection statistics. The fluctuations at the left and right contacts are assumed to be uncorrelated as well.

\section{A. Injected electron-density fluctuations}

The electron-density fluctuations at a slice $x$ caused by stochastic injection from the contacts is obtained by summing up all the contributions (A15) derived in the Appendix. In terms of the injected current fluctuations (58), we obtain the following expression:

$$
\begin{aligned}
\delta n^{i n j}(x)= & \frac{1}{2 \sqrt{\pi} I_{c}} \sum_{k=L, R} \int_{\psi_{k}-\psi_{m}}^{\infty} \frac{\delta I_{k}(\varepsilon) d \varepsilon}{\sqrt{\varepsilon+\psi(x)-\psi_{k}}} \\
& +\frac{1}{\sqrt{\pi} I_{c}} \begin{cases}\int_{\psi_{L}-\psi(x)}^{\psi_{L}-\psi_{m}} \frac{\delta I_{L}(\varepsilon) d \varepsilon}{\sqrt{\varepsilon+\psi(x)-\psi_{L}}}, & 0<x<x_{m} \\
\int_{\psi_{R}-\psi(x)}^{\psi_{R}-\psi_{m}} \frac{\delta I_{R}(\varepsilon) d \varepsilon}{\sqrt{\varepsilon+\psi(x)-\psi_{R}}}, & x_{m}<x<\lambda .\end{cases}
\end{aligned}
$$

\section{B. Induced electron-density fluctuations}

The electron-density fluctuations induced at a slice $x$ by the fluctuations of the potential is obtained by summing up all the contributions (A16), and one gets

$$
\delta n^{i n d}(x)=n(x) \delta \psi(x) \pm \frac{J}{2 \sqrt{\pi} w_{*}(x)}\left[\delta \psi(x)-\delta \psi_{m}\right] \text {, }
$$

where $J \equiv \bar{I} / I_{c}$, and the upper sign applies in the interval $0<x<x_{m}$ and the lower sign applies in the interval $x_{m}<x<\lambda$. This term along with the term (60) should then be used in the perturbed Poisson equation.

\section{Current fluctuations}

The expression for the fluctuation of the current in any section of the sample is given by

$$
\delta I=2 \sqrt{\pi} I_{c} \int_{-\infty}^{\infty} \delta f(x, w) w d w .
$$

Now we have to substitute into Eq. (62) the fluctuation of the distribution function, which is convenient to consider here as a sum of the homogeneous and nonhomogeneous parts of the solution of the kinetic equation [see Eq. (A4) in Appendix]. The contribution of the nonhomogeneous term is zero, which can be easily checked by direct integration of Eq. (A7). The homogeneous term consists of the transmitted and reflected parts given by Eqs. (A6). Again, the reflected electrons give zero contribution to the current fluctuations, since the functions (A6b) are even on $w$, so that the integrand (62) is an odd function and its integration from $-\infty$ to $\infty$ yields zero. The only nonzero contribution comes from the terms (A6a) for transmitted perturbing electrons. Substituting them into Eq. (62) and changing the variable of integration from $w$ to $w_{c}$, we obtain

$$
\begin{aligned}
\delta I= & 2 \sqrt{\pi} I_{c}\left[\int_{w_{L}}^{\infty} \delta f_{L}\left(w_{c}\right) w_{c} d w_{c}+\int_{-\infty}^{-w_{R}} \delta f_{R}\left(w_{c}\right) w_{c} d w_{c}\right] \\
& +I_{c} e^{\psi_{m}}\left(e^{-\psi_{L}}-e^{-\psi_{R}}\right) \delta \psi_{m}
\end{aligned}
$$

from which it is seen that the current fluctuation is independent of the position $x$. By using the definition for the injected current fluctuation (58) and the formula (47) for the average current, the final expression for the current fluctuation takes on the form 


$$
\delta I=\int_{V_{m}}^{\infty} \delta I_{L}(\varepsilon) d \varepsilon-\int_{V_{m}+V}^{\infty} \delta I_{R}(\varepsilon) d \varepsilon-\bar{I} \delta V_{m},
$$

where $\delta V_{m} \equiv-\delta \psi\left(x_{m}\right)$ is the potential minimum fluctuation. $\delta I$ depends on the magnitude of the fluctuating potential barrier irrespective of its random location. This is a consequence of the current conservation along the diode.

Equation (64) is a central one, which determines the fluctuation of the transmitted current through the fluctuations injected from the contacts. The first two terms in the rhs represent the current fluctuations transmitted directly to the opposite contact from the left and right contacts, respectively. Since the injected electrons of different energies are uncorrelated, they give the full shot noise. It is the last term $-\bar{I} \delta V_{m}$, caused by the self-consistent potential fluctuation (long-range Coulomb correlations), that compensates the current fluctuation and may result in the noise reduction. We note, first, that it is proportional to the current and thus exists only under nonequilibrium conditions. Second, it depends on the potential barrier fluctuation $\delta V_{m}$. When the barrier does not appear under certain conditions, all the injected fluctuations are transmitted to the opposite contact and the noise of the transmitted current is expected to be the same as that for the injected carriers, i.e., the full Poissonian shot noise. The compensating behavior may occur only when the potential barrier is present. Notice that the contributions of the leftand right-injected fluctuations are of the opposite sign, i.e., $\delta I_{L}>0$ increases the fluctuation of the transmitted current, while $\delta I_{R}>0$ decreases it.

Among all the injecting perturbing electrons, only those able to pass over the potential barrier contribute to the transmitted current fluctuation. This fact is reflected in the lower integration limits that contain the height of the potential barrier. In constrast, all the injected electrons contribute to the potential barrier fluctuations, and thereby participate in the compensation effect, as it will be shown in the next section.

\section{Self-consistent potential fluctuations}

We find the potential barrier fluctuation $\delta V_{m}$, which is of prime interest, from the linearized Poisson equation (56) for the potential fluctuations $\delta \psi$. By substituting the electrondensity fluctuations $\delta n$ consisting of the injected and induced contributions found in Secs. V A and V B, we obtain for the self-consistent potential fluctuations

$$
\begin{aligned}
\frac{d^{2} \delta \psi}{d x^{2}} & =\delta n^{i n d}(x)+\delta n^{i n j}(x) \\
& =n(x) \delta \psi(x) \pm \frac{J}{2 \sqrt{\pi} w_{*}(x)}\left[\delta \psi(x)-\delta \psi_{m}\right]+\delta n^{i n j}(x) .
\end{aligned}
$$

This is a second-order nonhomogeneous differential equation with spatially dependent coefficients, where the term $\delta n_{x}^{i n j}$, dependent on the fluctuations at the contacts $\delta I_{k}$ [see Eq. (60)], plays the role of a stochastic noise source. To find its solution in a general form is a complicated problem. In addition, we remark that the term with $1 / w_{*}(x)$ is singular at the potential barrier minimum $x=x_{m}$ which produces an ad- ditional difficulty. Nevertheless, we will show that it can be solved exactly without any approximation. First of all, it is advantageous to introduce a new stochastic quantity

$$
\delta \eta_{x}=\delta \psi(x)-\delta \psi_{m},
$$

which is the potential fluctuation at a slice $x$ measured from the fluctuating potential minimum. Thus, due to our choice, at the potential minimum $\delta \eta_{x_{m}}=0$, where $x_{m}=x_{m}^{0}+\delta x_{m}$ is a stochastic location of the potential minimum fluctuating around its steady-state position $x_{m}^{0}$. The latter fluctuation, however, may be neglected, since it is only of second order in respect to the potential fluctuations, because of the property of the minimum $\psi^{\prime}\left(x_{m}^{0}\right)=0$. Thus, one gets the stochastic differential equation

$$
\begin{aligned}
\hat{L} \delta \eta_{x} & \equiv\left[\frac{d^{2}}{d x^{2}}-n(x) \mp \frac{J}{\sqrt{4 \pi \eta(x)}}\right] \delta \eta_{x} \\
& =-n(x) \delta \eta_{L}+\delta n^{i n j}(x) .
\end{aligned}
$$

The boundary conditions for this equation follows from Eqs. (57) and (66):

$$
\delta \eta_{L}=\delta \eta_{R}=-\delta \psi_{m} .
$$

Since the potential $\delta \eta_{x}$ is referenced to the fluctuating minimum, its values on the contacts are not zero, while in a stationary frame they are zero due to a fixed-applied-voltage conditions.

To find the solution of Eq. (67), we use a method we have recently applied for a stochastic drift-diffusion equation which has a similar form. ${ }^{52}$ Essentially, this method is based on the possibility of finding two (arbitrary) linearly independent solutions of the corresponding homogeneous equation $\hat{L} \delta \eta_{x}=0$, which can further be used to construct the solution for the nonhomogeneous equation satisfying the appropriate boundary conditions. One of the solutions is proportional to $(d \psi / d x)$, which can be seen by differentiating the Poisson equation (21) and comparing the result with Eq. (67) with zero rhs. For convenience, we take it as $E(x)=-(d \psi / d x)$ so the solution coincides with the electric field profile. In general, ${ }^{52}$ the second solution can be obtained from the first one by using the formula $u(x)=E(x) \int_{C}^{x}\left[W(y) / E^{2}(y)\right] d y$, where $W(x)=E(x) u^{\prime}(x)-E^{\prime}(x) u(x)$ is the Wronskian, $C$ is an arbitrary constant, the prime stands for the derivative, and $E(x) \neq 0, \forall x$ is assumed. However, this formula cannot be applied for our problem, since $E(x)=0$ precisely at the point of the potential minimum and the integral diverges. Alternatively, we use another formula for the second solution $u$ which has no divergence in the whole region. Explicitly,

$$
u(x)=-\frac{W(x)}{E^{\prime}(x)}+E(x) \int_{C}^{x} \frac{W(y) Q(y)}{\left[E^{\prime}(y)\right]^{2}} d y,
$$

where the function $Q(x)=-n(x) \mp J / \sqrt{4 \pi \eta(x)}$ is a free term in the operator $\hat{L}$, and the necessary condition $E^{\prime}(x)$ $\neq 0$ is fulfilled. Next we notice that the differential operator $\hat{L}$ given by Eq. (67) does not contain the term with the first derivative, which leads to the constant Wronskian. The value of this constant is not actually important, since it will be canceled as will be seen below, so we take $W(x)=1$. The 
arbitrary constant $C$ in Eq. (69) does not influence the final results. It is convenient, however, to define it by the conditions $u(0)=u(\lambda)=0$ at the ends of the diode, which correspond to the homogeneous boundary conditions for the Green functions of the operator $\hat{L}$ and provide the most compact intermediate expressions. To satisfy the zero boundary conditions on both ends of the diode, one can take the function $u(x)$ as consisting of two branches. As a result, we obtain the following expression:

$$
\begin{aligned}
& u(x)=\frac{1}{n(x)}+E(x)
\end{aligned}
$$

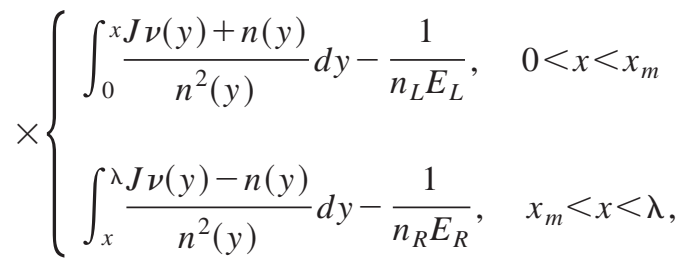

where $\nu(x) \equiv 1 / \sqrt{4 \pi \eta(x)}$ and $n(x)$ and $E(x)$ are the steadystate spatial profiles of the electron density and electric field, which take the values at the left and right contacts $n_{L}, E_{L}$ and $n_{R}, E_{R}$, respectively. The function $u(x) \geqslant 0$ is continuous in the entire region $0<x<\lambda$, including the point of the potential minimum, where it takes the value $u\left(x_{m}\right)=1 / n_{m}$. At that point, however, it has an infinite derivative, which is a consequence of the zero of the field.

The general solution of Eq. (67), satisfying the boundary conditions (68) and the conditions $E\left(x_{m}\right)=0, \delta \eta_{x_{m}}=0$, then reads

$$
\begin{aligned}
\delta \eta_{x}= & E(x) \int_{0}^{x} u(y) \delta s_{y} d y+u(x) \int_{x}^{x_{m}} E(y) \delta s_{y} d y \\
& +\delta \eta_{L} \frac{E(x)}{E_{L}}, \quad 0<x<x_{m}, \\
\delta \eta_{x}= & -E(x) \int_{x}^{\lambda} u(y) \delta s_{y} d y-u(x) \int_{x_{m}}^{x} E(y) \delta s_{y} d y \\
& +\delta \eta_{L} \frac{E(x)}{E_{R}}, \quad x_{m}<x<\lambda,
\end{aligned}
$$

where $\delta s_{x}=n(x) \delta \eta_{L}-\delta n^{i n j}(x)$ is the nonhomogeneous part of Eq. (67). Thus, one can find the potential fluctuation $\delta \eta_{x}$ at any section $x$ of the sample. In particular, its value at the boundaries yields the potential barrier fluctuation $\delta V_{m}$ $=\delta \eta_{L}$. We find the unknown $\delta \eta_{L}$ from the continuity condition on the derivative $d \delta \eta / d x$ at $x=x_{m}$ :

$$
\delta \eta_{L}\left[\frac{1}{E_{R}}-\frac{1}{E_{L}}\right]=\int_{0}^{\lambda} u(x) \delta s_{x} d x .
$$

Now recalling that $\delta \eta_{L}$ has entered also in $\delta s$, we obtain

$$
\delta V_{m}=\frac{1}{\Delta} \int_{0}^{\lambda} u(x) \delta n^{i n j}(x) d x
$$

with

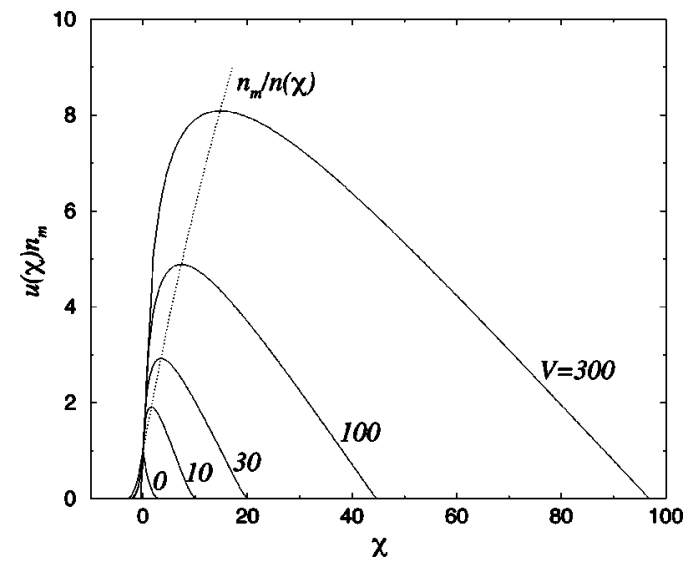

FIG. 8. Function $u(\chi)$ which shows the relative contributions of the noise sources $\delta n^{i n j}(\chi)$ to the potential barrier fluctuations for $\lambda=100$ and for several biases $V$. The potential minimum is located at $\chi=0$.

$$
\Delta=\frac{1}{E_{L}}-\frac{1}{E_{R}}+\int_{0}^{\lambda} u(x) n(x) d x .
$$

The last integral can further be reduced by substituting $n=$ $-d E / d x$ and the expression for $u(x)$ given by Eq. (70). Integrating by parts, one gets the simple formula

$$
\Delta=\frac{\lambda}{2}+\frac{1}{E_{L}}-\frac{1}{E_{R}} .
$$

The obtained analytical expression (73) with the parameter $\Delta$ given by Eq. (75) yields the fluctuation of the barrier height in terms of the spatially distributed "noise source" $\delta n^{i n j}(x)$ caused by the random injection from the contacts. The weight function $u(x)$ shows the relative contributions of the "noise sources" to the potential barrier fluctuations. Its behavior is illustrated in Fig. 8, where we present $u$ normalized to $1 / n_{m}$ as a function of the coordinate $\chi=\left(x-x_{m}\right) / l_{m}$. In such a scaling for a fixed voltage, $u(\chi) n_{m}$ is almost independent of $\lambda$ with a slight deviation at the ends of the function extension. An interesting property of those functions for different biases is that they cross the curve $n_{m} / n(\chi)$ (the inverse universal density profile as discussed in Sec. IV E) at two characteristic points: the potential minimum $\chi=0$ where $d u / d \chi=\infty$, and at the maximum of $u(\chi)$ (see Fig. 8). The latter point has significance in that the electron-density fluctuations there have the largest influence on the potentialbarrier fluctuations. It is worth noting that the maximum contribution to $\delta V_{m}$ does not come from the potential minimum location, as it would seem intuitively.

\section{E. Current noise spectral density}

Substituting the obtained formula (73) for $\delta V_{m}$ into Eq. (64), we obtain the current fluctuation as

$$
\delta I=\int_{0}^{\infty} \gamma_{L}(\varepsilon) \delta I_{L}(\varepsilon) d \varepsilon+\int_{0}^{\infty} \gamma_{R}(\varepsilon) \delta I_{R}(\varepsilon) d \varepsilon,
$$




$$
\begin{aligned}
& \gamma_{L}(\varepsilon)=\left\{\begin{array}{l}
-2 J \int_{0}^{x_{L}^{*}} K(x, \varepsilon) d x, \quad \varepsilon<V_{m} \\
1-J \int_{0}^{\lambda} K(x, \varepsilon) d x, \quad \varepsilon>V_{m},
\end{array}\right. \\
& \gamma_{R}(\varepsilon)=\left\{\begin{array}{l}
-2 J \int_{x_{R}^{*}}^{\lambda} K(x, \varepsilon-V) d x, \quad \varepsilon<V_{m}+V \\
-1-J \int_{0}^{\lambda} K(x, \varepsilon-V) d x, \quad \varepsilon>V_{m}+V,
\end{array}\right.
\end{aligned}
$$

where $K(x, \varepsilon)=u(x) /[2 \sqrt{\pi} \Delta \sqrt{\varepsilon+\psi(x)}]$, and $x_{L}^{*}$ and $x_{R}^{*}$ are found from $\varepsilon=-\psi\left(x_{L}^{*}\right)=V-\psi\left(x_{R}^{*}\right)$. The functions $\gamma_{k}(\varepsilon)$ introduced for each contact have the meaning of current fluctuation transfer functions, since they represent the ratio of the transmitted current fluctuation to the injected current fluctuation for a particular injection energy $\varepsilon$. The terms proportional to the current $J$ originate from the potential minimum fluctuations, whereas the constant contributions $( \pm 1)$ represent the direct transmission of fluctuations to the opposite contact.

Equation (76) leads to the spectral density of current fluctuations

$$
S_{I}=2 q I_{c} \int_{0}^{\infty}\left[\gamma_{L}^{2}(\varepsilon)+\gamma_{R}^{2}(\varepsilon)\right] e^{-\varepsilon} d \varepsilon
$$

This equation with $\gamma_{k}(\varepsilon)$ given by formulas (77) and (78) is the final result of our derivations. It allows us to obtain the current-noise spectral density, for the given level of screening $\lambda$ and applied voltage $V$, from the steady-state distributions of the potential $\psi(x)$, electric field $E(x)$, and electron density $n(x)$ by direct integration. Thus, the current-noise level is directly related to the transport inhomogeneity in the system. Note that the obtained formulas are exact for biases ranging from thermal to shot-noise limits under a spacecharge-limited transport conditions.

For practical calculations of the transfer functions $\gamma_{k}(\varepsilon)$, one may integrate by parts the function $K$ in formulas (77) and (78), which leads to the following expressions corresponding to each group of carriers:

$$
\begin{array}{rlrl}
\gamma_{L, r}(\tilde{\varepsilon})= & -\frac{\beta}{2 \Delta_{m}} \int_{-\tilde{\varepsilon}\left[h_{V}^{-}(\eta)\right]^{3 / 2}}^{\eta_{L}} \frac{G(\eta, \tilde{\varepsilon}),}{} & & \tilde{\varepsilon}<0, \\
\gamma_{L, t}(\tilde{\varepsilon})=1-\frac{\beta}{2 \Delta_{m}}\left\{\int_{0}^{\eta_{L}} \frac{H(\eta, \tilde{\varepsilon})}{\left[h_{V}^{-}(\eta)\right]^{3 / 2}} d \eta\right. & & \\
& \left.+\int_{0}^{\eta_{R}} \frac{H(\eta, \tilde{\varepsilon})}{\left[h_{V}^{+}(\eta)\right]^{3 / 2}} d \eta\right\}, & & \tilde{\varepsilon}>0, \\
\gamma_{R, r}(\tilde{\varepsilon})= & -\frac{\beta}{2 \Delta_{m}} \int_{V-\tilde{\varepsilon}}^{\eta_{R}} \frac{G(\eta, \tilde{\varepsilon}-V)}{\left[h_{V}^{+}(\eta)\right]^{3 / 2}} d \eta, & \tilde{\varepsilon}<0, \\
\gamma_{R, t}(\tilde{\varepsilon})=\gamma_{L, t}(\tilde{\varepsilon}-V)-2, &
\end{array}
$$

where $\tilde{\varepsilon}=\varepsilon-V_{m}$ is the injection electron energy referenced from the potential minimum,

$$
\begin{gathered}
\Delta_{m} \equiv \frac{\Delta}{l_{m}}=\frac{\lambda_{m}}{2}+\frac{1}{\sqrt{h_{V}^{-}\left(\eta_{L}\right)}}+\frac{1}{\sqrt{h_{V}^{+}\left(\eta_{R}\right)}}, \\
H(\eta, \widetilde{\varepsilon}) \equiv \frac{2}{\sqrt{\pi}}[\sqrt{\eta+\widetilde{\varepsilon}}-\sqrt{\tilde{\varepsilon}}] \\
G(\eta, \tilde{\varepsilon}) \equiv \frac{4}{\sqrt{\pi}} \sqrt{\eta+\widetilde{\varepsilon}}
\end{gathered}
$$

Formulas (80a) and (80b) with $\beta=1$ correspond to the formulas for a vacuum diode found by North within different approach [see Eqs. (31) and (38) of Ref. 8].

\section{F. Nyquist equilibrium noise}

In equilibrium, $\bar{I} \rightarrow 0$, the compensating term $\bar{I} \delta V_{m}$ in Eq. (64) vanishes, and, comparing with Eq. (76), the transfer functions are simply the step functions with a step at the barrier height: $\gamma_{L}^{e q}(\varepsilon)=\theta\left(\varepsilon-V_{m}\right), \quad \gamma_{R}^{e q}(\varepsilon)=-\theta\left(\varepsilon-V_{m}\right)$. This means that only electrons able to pass over the barrier contribute to the equilibrium (thermal) noise. For this case, one can easily obtain the Nyquist noise formula

$$
S_{I}^{e q}=4 q I_{c} e^{-V_{m}^{0}}=4 k_{B} T g_{0},
$$

where $g_{0}=d \bar{I} /\left.d U\right|_{U \rightarrow 0}$ is the zero-bias small-signal conductance. [To find the conductance we have made use of Eq. (47).] Both electron streams, from the left and right contacts, equally contribute to the Nyquist noise. The space-charge effect on the equilibrium noise is present in the dependence of $g_{0}$ on the potential minimum $V_{m}$.

\section{G. Noise-reduction factor}

The obtained formula (73) for the current-noise spectral density $S_{I}$, which accounts for the long-range Coulomb correlations, may be compared with the uncorrelated value through the so-called noise-reduction factor. Out of equilibrium, if one neglects the term $I \delta V_{m}$ in Eq. (64), which is responsible for the long-range Coulomb correlations between the carriers, one obtains $\gamma_{L}^{\text {uncor }}(\varepsilon)=\theta\left(\varepsilon-V_{m}\right), \gamma_{R}^{\text {uncor }}(\varepsilon)$ $=-\theta\left(\varepsilon-V_{m}-V\right)$, which leads to

$$
\begin{aligned}
S_{I}^{\text {uncor }}=2 q\left(I_{L R}+I_{R L}\right) & =2 q \bar{I} \operatorname{coth}(V / 2) \\
& \approx 2 q \bar{I}, \quad V \gtrsim 5,
\end{aligned}
$$

which is nothing more than the Poissonian noise of two uncorrelated streams of carriers opposite each other (at high voltages the contribution from the right-contact stream becomes negligible). It is reasonable, therefore, to define the noise-reduction factor by

$$
\Gamma=\frac{S_{I}}{S_{I}^{\text {uncor }}}=\frac{S_{I}}{2 q \bar{I} \operatorname{coth}(V / 2)} .
$$

By this definition, both the thermal noise and shot noise limits are included. ${ }^{5}$ 


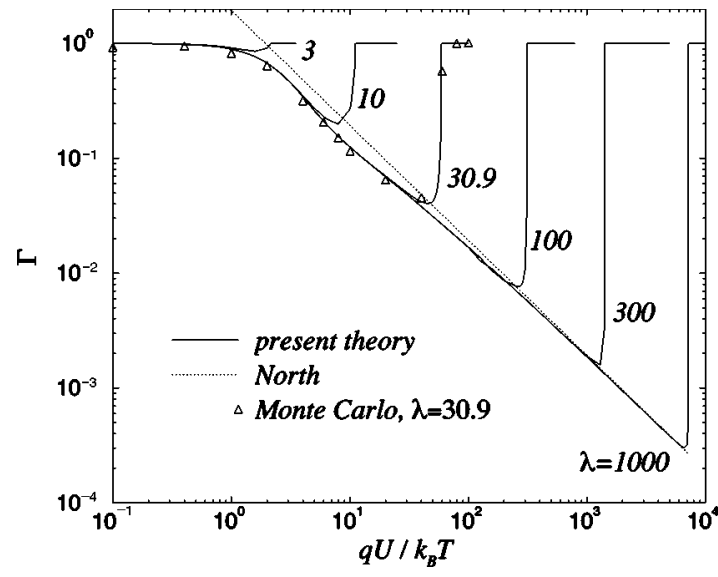

FIG. 9. Current-noise reduction factor $\Gamma$ vs bias $U$ for different levels of screening $\lambda=d / L_{D}^{0}$ (solid). For comparison, North's asymptotic solution given by Eq. (89) is shown (dots). For the case of $\lambda=30.9$, the results are shown to be in excellent agreement with the Monte Carlo simulations (Ref. 5) (triangles).

Figure 9 shows $\Gamma$ versus applied voltage $V$ for various screening parameters $\lambda$. At low values of $\lambda$, the noisereduction effect is weak, $\Gamma \approx 1$. As $\lambda$ increases, the noise becomes substantially reduced in the range of biases $k_{B} T$ $\lesssim q U<q U_{c r}$, where $U_{c r}$ is a critical voltage for which the potential minimum vanishes (its value is a function of $\lambda$ ). At $U \geqslant U_{c r}$ the full shot-noise level is abruptly recovered. This sharp increase in the noise intensity when observed in an experiment would indicate on the disappearance of the potential barrier controlling the current.

We have compared our results for the noise reduction factor with those obtained by the Monte Carlo simulations. ${ }^{5}$ The agreement was found to be perfect within numerical uncertainty of the Monte Carlo algorithm, as it is seen from Fig. 9 where we show such a comparison for $\lambda=30.9$. The agreement for the noise characteristics, as well as for the steady-state spatial profiles and $I-V$ curves, indicates the correspondence between our kinetic theory and the Monte Carlo model used in Refs. 5, 6, and 48.

An advantage of our analytical approach is that, in addition to the net noise characteristics, one may distinguish the relative contributions to the noise from different groups of carriers. In Fig. 10 we present the results for the noisereduction factor $\Gamma$ as a sum of four contributions. It is seen that in equilibrium only the transmitted electrons contribute to the noise (equally from the left and right contacts). In the range $1 \leqq V \lesssim 10$, the contribution from the reflected carriers becomes appreciable with a maximum at $V \approx 3$. At higher voltages, as the potential barrier progressively decreases, the role of the reflected carriers becomes less important. The contribution of the right-contact transmitted electrons is negligible at $V \gtrsim 5$, as for the stationary $I-V$ characteristics. As a result, in the high-voltage limit, only the left-contact transmitted electrons contribute to the noise. This fact can be taken into account in analyzing the asymptotic behavior of the noise-reduction factor at high-voltage limit. In this limit the main contribution to the current fluctuation transfer function comes from $\gamma_{L, t}$. Under the condition $V_{m} \ll V<V_{c r}$, which is easy to satisfy at large $\lambda$, the first integral in Eq. (80b) is much less than the second one, so that the contribu-

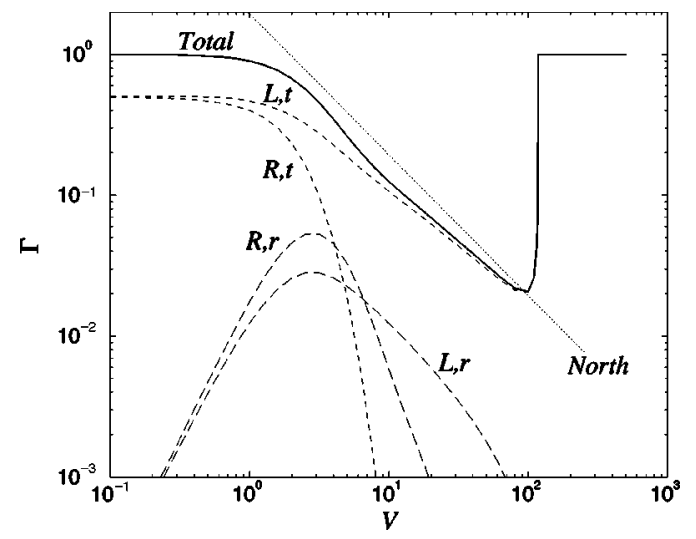

FIG. 10. Contributions to the current-noise-reduction factor $\Gamma$ corresponding to different electron groups for the case of $\lambda=50 . L$ and $R$ refer to the left and right contacts, and $t$ and $r$ distinguish the transmitted and reflected groups of carriers. North's asymptotic solution is shown by dots.

tion to the noise from the region before the virtual cathode may be neglected. Furthermore, at sufficiently high $\lambda, \Delta_{m}$ $\approx \frac{1}{2} \lambda / l_{m} \approx \frac{1}{2} \lambda \sqrt{J}$. Thus, one can write

$$
\begin{aligned}
\gamma_{L, t}(\tilde{\varepsilon}) & \approx 1-\frac{1}{\lambda \sqrt{J}} \int_{0}^{V} \frac{H(\eta, \tilde{\varepsilon})}{\left[h_{V}^{+}(\eta)\right]^{3 / 2}} d \eta \\
& \approx 1-\frac{\pi^{1 / 4} 2^{3 / 2} V^{3 / 4}}{\lambda \sqrt{J}}\left[\frac{1}{3}+\left(\frac{3}{4} \sqrt{\pi}-\sqrt{\tilde{\varepsilon}}\right) V^{-1 / 2}\right],
\end{aligned}
$$

where we have taken into account that the main contribution comes at the upper integration limit and made use of the asymptotic expansion of the function $h_{V}^{+}$given by Eq. (51). It is also assumed here that for any fixed energy the bias is high, $V \gg \widetilde{\varepsilon}$. It is justified since the range of valuable energies is limited by the Maxwellian exponentially decaying distribution. Now, substituting the Langmuir expression (53) for the current and neglecting $x_{m}$ and $V_{m}$, one obtains

$$
\gamma_{L, t}(\tilde{\varepsilon}) \approx \frac{3}{\sqrt{V}}\left(\sqrt{\tilde{\varepsilon}}-\frac{\sqrt{\pi}}{2}\right) .
$$

This formula, after the integration over the energies, leads to North's asymptotic formula ${ }^{8}$ for the noise-reduction factor:

$$
\Gamma \approx \frac{9}{V}\left(1-\frac{\pi}{4}\right) \approx \frac{1.9314}{V}, \quad V \rightarrow \infty .
$$

This formula is universal in the sense that it is free from any diode parameter including the screening parameter $\lambda$. However, it is assumed that $\lambda$ should be sufficiently high to satisfy the simultaneous conditions $V \rightarrow \infty$ and $V<V_{c r}$. As it is seen from Fig. 9, the noise-reduction factor $\Gamma$ approaches this asymptotic formula at high values of the parameters: $\lambda$ $\gtrsim 10^{3}, \quad V \gtrsim 10^{3}$. As we have already noted, in semiconductors it is hard to maintain the ballistic regime at biases $V$ $\gtrsim 50$ because of the increasing significance of electronphonon interactions, which destroy the ballistic regime. In the range of interest $1 \leqq V \lesssim 50$ the noise level is seen to be significantly lower than North's asymptotic curve. This 


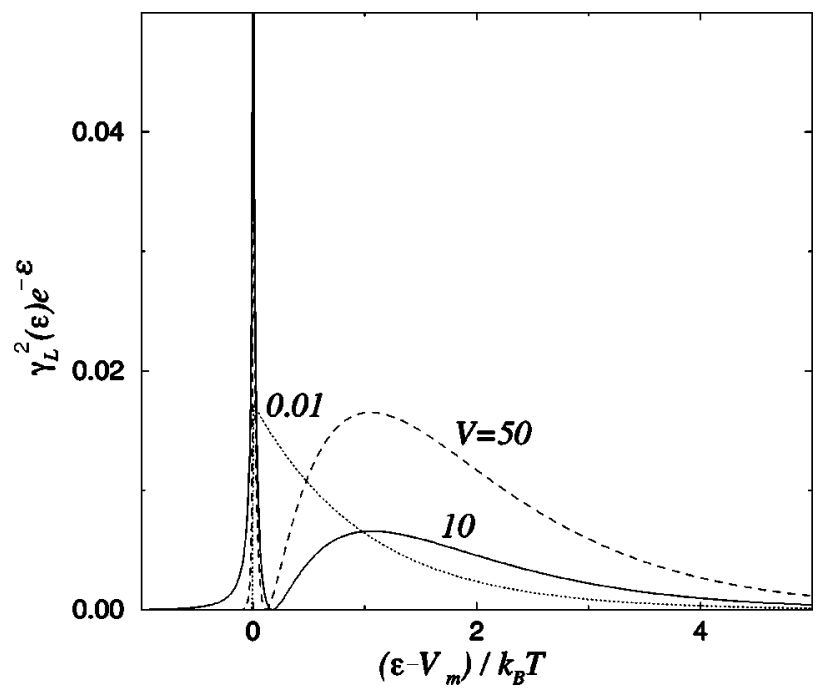

FIG. 11. Partial contributions to the current-noise spectral density from different energies $\varepsilon$ of electrons injected from the left contact for biases $V=0.01 ; 10 ; 50$. The results for the rightcontact electrons are approximately the same for $V=0.01$ and negligibly small for $V=10$ and 50 .

means that the full set of formulas are necessary to describe properly the noise intensity in the semiconductor ballistic diodes. Another important conclusion from Fig. 9 is that for a nondegenerate electron gas there exists the lowest noisereduction level dependent only on the bias and the temperature through the factor $q U /\left(k_{B} T\right)$, and it is impossible to surmount it by any choice of the material parameter and/or geometrical parameters of the diode. This universal minimalnoise curve approaches North's asymptotic curve at high voltages.

\section{H. Spectroscopy of shot noise}

A great advantage of the derived formula (79) for the current-noise spectral density is that one may obtain the partial contribution to the noise from electrons of different injection energies by computing the current fluctuation transfer functions $\gamma_{k}(\varepsilon)$. The electrons for which $\gamma_{k}(\varepsilon)<0$ reduce the current fluctuations. For instance, the right-contact electrons always reduce them, since $\gamma_{R}(\varepsilon)<0, \forall \varepsilon$. The reflected carriers originated from the left contact $\left(\varepsilon<V_{m}\right)$ also provide negative values for the transfer function and compensate the current fluctuations by virtue of the potentialbarrier fluctuations. The same effect is produced by the leftcontact transmitted electrons with the energies slightly above the barrier height $V_{m}$. From both groups, the most efficient compensation carriers are those with energies in the vicinity of $V_{m}$ where $\gamma_{L} \rightarrow-\infty .{ }^{53}$ They provide an overcompensation of the injected from the contacts fluctuations. In constrast, the injected electrons whose energy greatly exceeds $V_{m}$ produce negligible perturbations of the potential barrier, thus leading to the asymptotic behavior $\gamma_{L}(\varepsilon) \rightarrow 1, \gamma_{R}(\varepsilon) \rightarrow-1$ as $\varepsilon \rightarrow \infty$. There also exists the specific energy $\varepsilon^{*}$, for which the compensation fluctuation is exactly equal to the injected fluctuation, giving no noise at all, $\gamma_{L}\left(\varepsilon^{*}\right)=0$. This curious fact is illustrated in Fig. 11 where we present the contribution to the current-noise spectral density from different energies of electrons injected from the left contact by plotting the function $\gamma_{L}^{2}(\varepsilon) e^{-\varepsilon}$. At high biases, just after the peak at $\varepsilon$ $=V_{m}$, the point with zero contribution to the noise is observed. While at equilibrium the maximum contribution comes from the carriers injected with $\varepsilon=V_{m}$, at high biases, when the noise reduction is significant, the main contribution comes from the electrons that are injected above the potential barrier height by the value about $k_{B} T$. Therefore, the integral noise-reduction effect is a consequence of the suppression of the contributions from the electron energies in the vicinity of $\varepsilon^{*}$.

The obtained exact solutions allows us to investigate in great detail the correlations between different groups of carriers. While the injected carriers are uncorrelated, those in the volume of the conductor are strongly correlated, as follows from the derived formulas for the fluctuation of the distribution function [see general expressions (A12) and (A13) in the Appendix]. Those correlations may be observed experimentally by making use of a combination of two already realized techniques: a hot-electron spectrometer ${ }^{50,51}$ and shot-noise measurements. ${ }^{34-36}$ The electron spectrometer, placed behind the receiving semitransparent contact, acts as an analyzer of electron distribution over the energy. ${ }^{50,51}$ In this way spectroscopic information, that is, the average partial currents $\bar{I}(\widetilde{\varepsilon})$ and their fluctuations $\delta I(\widetilde{\varepsilon})$, may be measured for different energies $\tilde{\varepsilon}$ of electrons collected at the contact. This is similar to the energy-resolved noise measurements realized in Ref. 54. The partial current of the transmitted electrons at the receiving (right) contact is given by $\bar{I}(\tilde{\varepsilon})=I_{c} e^{-\tilde{\varepsilon}-V_{m}} \theta(\tilde{\varepsilon})$, where the threshold energy $\tilde{\varepsilon}=0$ corresponds to the arriving electrons that have a zero longitudinal kinetic energy at the potential minimum. To find the fluctuation $\delta I(\tilde{\varepsilon})$, we consider the fluctuation of the distribution function $\delta f(x, w)$ at $x=\lambda$. Since $\delta \psi(\lambda)=0$, the terms with $\delta \psi(x)$ vanish. Thus, for the transmitted over the barrier electrons which contribute to the current, from Eqs. (A12a) and (A13a) one obtains

$$
\begin{aligned}
\delta f_{L, t}(\lambda, w)= & \delta f_{L}(\lambda, w) \theta\left(w-w_{R}\right) \\
& -\frac{1}{\sqrt{\pi}} e^{-w^{2}+V} \delta V_{m} \frac{1}{2 w} \delta\left(w-w_{R}\right) .
\end{aligned}
$$

Since only the positive velocities are considered, one can change the velocity variable to the energy by $\tilde{\varepsilon}=w^{2}-w_{R}^{2}$, and obtain

$$
\delta f_{L, t}(\tilde{\varepsilon})=\delta f_{L}\left(\tilde{\varepsilon}+V_{m}\right) \theta(\tilde{\varepsilon})-\frac{1}{\sqrt{\pi}} e^{-\tilde{\varepsilon}-V_{m}} \delta V_{m} \delta(\tilde{\varepsilon}) .
$$

By using the relation (58) between the fluctuation of the contact distribution function and that of the contact injection current, we obtain

$$
\delta I(\tilde{\varepsilon})=\delta I_{L}\left(\tilde{\varepsilon}+V_{m}\right) \theta(\tilde{\varepsilon})-I_{c} e^{-V_{m}} \delta V_{m} \delta(\tilde{\varepsilon}) .
$$

Thus, the correlator for the current fluctuations becomes 


$$
\begin{aligned}
\left.\left\langle\delta I(\tilde{\varepsilon}) \delta I\left(\tilde{\varepsilon}^{\prime}\right)\right\rangle\right|_{x=\lambda}= & \left\langle\delta I_{L}\left(\tilde{\varepsilon}+V_{m}\right) \delta I_{L}\left(\tilde{\varepsilon}^{\prime}+V_{m}\right)\right\rangle \\
& -I_{c} e^{-V_{m}} \delta\left(\widetilde{\varepsilon^{\prime}}\right)\left\langle\delta I_{L}\left(\tilde{\varepsilon}+V_{m}\right) \delta V_{m}\right\rangle \\
& -I_{c} e^{-V_{m}} \delta(\tilde{\varepsilon})\left\langle\delta I_{L}\left(\tilde{\varepsilon}^{\prime}+V_{m}\right) \delta V_{m}\right\rangle \\
& +I_{c}^{2} e^{-2 V_{m}} \delta(\tilde{\varepsilon}) \delta\left(\tilde{\varepsilon}^{\prime}\right)\left\langle\delta V_{m}^{2}\right\rangle,
\end{aligned}
$$

where the average is taken over the injected fluctuations. It is clear that for $\widetilde{\varepsilon}, \widetilde{\varepsilon}^{\prime}>0$ the carriers remain uncorrelated since only the first term does not vanish. It is $\propto \delta\left(\tilde{\varepsilon}-\widetilde{\varepsilon}^{\prime}\right)$ due to the imposed injection conditions that should lead to the full shot noise. In such a case, an interesting question arises: What is the reason for the noise reduction obtained for the total (integrated over the energies) current fluctuations? The answer is found looking at the electrons with energies close to the threshold energy $\tilde{\varepsilon}=0$ ("tangent", electrons). All other electrons are anticorrelated with that group. This means that if there is a positive fluctuation of overbarrier electrons, there should be a negative one for the "tangent" electrons and vice versa. This anticorrelation explains the overall noise reduction. The tangent electrons can be thought as overcorrelated. The dispersion $\left\langle\delta I^{2}(\tilde{\varepsilon})\right\rangle$ has a sharp peak at $\tilde{\varepsilon}=0$ and then decreases with energy at $\tilde{\varepsilon}>0$. This peak is divergent ( $\delta$-shaped) in our collisionless theory. A small probability of scattering will lead to its broadening and finite magnitude. Therefore, by measuring the dispersion of the partial current fluctuations and/or their cross-correlations, one may observe a sharp peak and an anticorrelation of electrons, thus making the Coulomb correlations effect visible.

\section{SUMMARY}

In conclusion, we have presented a self-consistent theory of electron transport and noise in a ballistic two-terminal conductor under the conditions of nondegenerate electron gas. Our description is valid for ballistic electrons in solids as well as in vacuum. By solving analytically the kinetic equation coupled self-consistently with a Poisson equation, we have derived the electron distribution function and its fluctuation at arbitrary section $x$ of the conductor. This allowed us to obtain the steady-state spatial distributions of the transport characteristics, the $I-V$ curves, and the noise characteristics. While the time-averaged quantities are not affected by the Coulomb correlations, the noise characteristics are demonstrated to be drastically modified when those correlations are taken into account. Our results are in excellent agreement with the preceding Monte Carlo simulations. ${ }^{5,48}$

The obtained formulas have been analyzed in a wide range of biases and compared with the correspondent theory for the vacuum diode. In particular, we have demonstrated that the known formulas for vacuum electronics, such as the Child 3/2-power law for $I-V$ characteristics or North's asymptotic formula for the noise may not be applied for the semiconductor diode at biases that are relevant for the ballistic transport regime. Instead, one should use the more general formulas described in the present paper from which follows (i) the linear or sublinear $I-V$ dependences even under a strong limitation of transport by a space charge; (ii) a noise level significantly below the level obtained from North's formula; (iii) the sharp recovering of the full shot-noise level at a certain critical voltage; (iv) all the transport and noise characteristics determined by two dimensionless parameters: the screening parameter $\lambda=d / L_{D}^{0}$ and the bias $q U / k_{B} T$.

Based on the derived formula for the current-noise spectral density one may distinguish the relative contributions to the noise from different groups of carriers. We have analyzed the contributions coming from the transmitted and reflected groups of carriers, as well as the partial contributions to the noise from electrons of different injection energies. Finally, it should be noted that the analytical approach that we have presented in the paper may be extended and applied to various systems, e.g., for different kind of statistics of injecting electrons ${ }^{49}$ and other types of the contacts. On the other hand, the shot-noise suppression effect, which we treat analytically, may lead to important applications for low-noise small-size semiconductor devices, generators of subPoissonian light sources, ${ }^{55}$ etc. Our work then offers new perspectives on the study of Coulomb interactions and noise in small-size ballistic devices, such as ballistic transistors, point contacts, etc.

\section{ACKNOWLEDGMENTS}

We are grateful to T. González and L. Reggiani for fruitful collaboration on the Monte Carlo investigation of the present problem. This work has been partially supported by the Dirección General de Enseñanza Superior, Generalitat de Catalunya, Spain, and the NATO linkage grant HTECH.LG 974610.

\section{APPENDIX: FLUCTUATIONS OF THE DISTRIBUTION FUNCTION AND ELECTRON DENSITY}

In a similar way to the subdivision of the stationary distribution function (24) into the components corresponding to different groups of electrons classified in Sec. IV A, the fluctuation $\delta f(x, w)$ may be expressed as

$$
\delta f=\delta f_{L, t}+\delta f_{L, r}+\delta f_{R, t}+\delta f_{R, r}
$$

The boundary conditions for these functions are obtained by perturbing the steady-state boundary conditions (25) and using $\partial w_{k} / \partial \psi_{m}=-\left(2 w_{k}\right)^{-1}$. One gets

$$
\begin{aligned}
\delta f_{L, t}\left(0, w_{c}\right)= & \delta f_{L}\left(w_{c}\right) \theta\left(w_{c}-w_{L}\right) \\
& +\frac{1}{2 w_{L}} f_{L}\left(w_{c}\right) \delta\left(w_{c}-w_{L}\right) \delta \psi_{m}
\end{aligned}
$$

$\delta f_{L, r}\left(0, w_{c}\right)=\delta f_{L}\left(w_{c}\right) \theta\left(w_{L}^{2}-w_{c}^{2}\right)-f_{L}\left(w_{c}\right) \delta\left(w_{L}^{2}-w_{c}^{2}\right) \delta \psi_{m}$,

$$
\begin{aligned}
\delta f_{R, t}\left(\lambda, w_{c}\right)= & \delta f_{R}\left(w_{c}\right) \theta\left(-w_{c}-w_{R}\right) \\
& -\frac{1}{2 w_{R}} f_{R}\left(w_{c}\right) \delta\left(-w_{c}-w_{R}\right) \delta \psi_{m},
\end{aligned}
$$

$$
\begin{aligned}
\delta f_{R, r}\left(\lambda, w_{c}\right)= & \delta f_{R}\left(w_{c}\right) \theta\left(w_{R}^{2}-w_{c}^{2}\right) \\
& -f_{R}\left(w_{c}\right) \delta\left(w_{R}^{2}-w_{c}^{2}\right) \delta \psi_{m},
\end{aligned}
$$


where the additional terms proportional to $\delta \psi_{m}$ describe the changes in the distribution functions due to the potential barrier variation.

Now we have to solve the perturbed kinetic equation (55), which may be rewritten as

$$
\left(w \frac{\partial}{\partial x}+\frac{1}{2} \frac{d \psi}{d x} \frac{\partial}{\partial w}\right) \delta f(x, w)=-\frac{1}{2} \frac{\partial f}{\partial w} \frac{d \delta \psi}{d x},
$$

where the rhs is supposed to be a given function (for this step of calculations). A general solution of this nonhomogeneous partial differential equation is a sum of a solution of the homogeneous problem and a particular solution of the nonhomogeneous problem. Explicitly,

$$
\delta f_{k, j}=\delta f_{k, j}^{h o m}+\delta f_{k, j}^{n h o m}, \quad k=L, R, \quad j=t, r .
$$

The solution for the homogeneous problem is determined by the boundary conditions (A2). By making use of the energy-conservation law (27), we make a replacement

$$
w_{c}=\operatorname{sgn}(w) \sqrt{w^{2}-\psi(x)+\psi_{k}}
$$

and obtain different contributions to $\delta f_{k, j}^{h o m}$ in the form

$$
\begin{aligned}
\delta f_{k, t}^{h o m}(x, w)= & \delta f_{k}(x, w) \theta\left( \pm w-w_{*}(x)\right) \\
& \pm \frac{1}{2 w} f_{k}(x, w) \delta\left( \pm w-w_{*}(x)\right) \delta \psi_{m}, \\
\delta f_{k, r}^{h o m}(x, w)= & \delta f_{k}(x, w) \theta\left(w_{*}^{2}(x)-w^{2}\right) \\
& -f_{k}(x, w) \delta\left(w_{*}^{2}(x)-w^{2}\right) \delta \psi_{m},
\end{aligned}
$$

where $\delta f_{L, r}^{h o m}$ and $\delta f_{R, r}^{h o m}$ are defined in the regions $0<x$ $<x_{m}$ and $x_{m}<x<\lambda$, respectively. The upper sign applies for $\delta f_{L, t}^{h o m}$ and the lower sign applies for $\delta f_{R, t}^{h o m}$, both terms valid in the whole range $0<x<\lambda$. The critical velocity $w_{*}(x)$ is given by Eq. (29).

The solution of the nonhomogeneous problem can easily be found through the steady-state distribution function $f\left(\varepsilon_{t}\right)=f\left(w^{2}-\psi(x)\right)$ in terms of the total energy $\varepsilon_{t}$ or, equivalently, in terms of the injection velocity $w_{c}$,

$$
\delta f_{k, j}^{n h o m}=-\frac{\partial f_{k, j}}{\partial \varepsilon_{t}} \delta \psi=-\frac{1}{2 w_{c}} \frac{\partial f_{k, j}}{\partial w_{c}} \delta \psi .
$$

Differentiating Eqs. (25), we find

$\delta f_{L, t}^{n h o m}=f_{L}\left(w_{c}\right) \delta \psi(x)\left[\theta\left(w_{c}-w_{L}\right)-\frac{1}{2 w_{c}} \delta\left(w_{c}-w_{L}\right)\right]$,

$\delta f_{R, t}^{n h o m}=f_{R}\left(w_{c}\right) \delta \psi(x)\left[\theta\left(-w_{c}-w_{R}\right)+\frac{1}{2 w_{c}} \delta\left(-w_{c}-w_{R}\right)\right]$,

$\delta f_{k, r}^{n h o m}=f_{k}\left(w_{c}\right) \delta \psi(x)\left[\theta\left(w_{k}^{2}-w_{c}^{2}\right)+\delta\left(w_{k}^{2}-w_{c}^{2}\right)\right]$.

In these equations the substitution (A5) is assumed, so that the fluctuations are finally the functions of $(x, w)$. Notice that the components for the reflected groups of carriers are defined in the regions: $0<x<x_{m}$ for $\delta f_{L, r}^{n h o m}$ and $x_{m}<x<\lambda$ for $\delta f_{R, r}^{n h o m}$, while those for the transmitted groups of carriers are given in the whole range $0<x<\lambda$.

According to the electrostatic boundary conditions (57) the fluctuations of the potential at the contacts are equal to zero, which leads to vanishing contributions (A8) at the contacts $\delta f^{n h o m}(0, w)=\delta f^{n h o m}(\lambda, w)=0$. The contributions (A6) satisfy the boundary conditions (A2). Thus, the distribution function in the form (A4) with eight contributions (A6) and (A8) is the solution of the problem for a given electrostatic potential $\psi(x)+\delta \psi(x)$.

For convenience of further consideration, we present $\delta f$ as a sum of the "injected" and "induced" contributions

$$
\delta f_{k, j}=\delta f_{k, j}^{i n j}+\delta f_{k, j}^{i n d}, \quad k=L, R, \quad j=t, r .
$$

In terms of the contact velocities $w_{c}$ (presented in such a form these equations will be frequently used throughout the paper), those contributions are given by

$\delta f_{k, t}^{i n j}\left(w_{c}\right)=\delta f_{k}\left(w_{c}\right) \theta\left( \pm w_{c}-w_{k}\right)$,

$\delta f_{k, r}^{i n j}\left(w_{c}\right)=\delta f_{k}\left(w_{c}\right) \theta\left(w_{k}^{2}-w_{c}^{2}\right)$,

and

$$
\begin{aligned}
\delta f_{k, t}^{i n d}\left(x, w_{c}\right)= & f_{k}\left(w_{c}\right)\left\{\theta\left( \pm w_{c}-w_{k}\right) \delta \psi(x)\right. \\
& \left.\mp \frac{1}{2 w_{c}} \delta\left( \pm w_{c}-w_{k}\right)\left[\delta \psi(x)-\delta \psi_{m}\right]\right\}, \\
\delta f_{k, r}^{i n d}\left(x, w_{c}\right)= & f_{k}\left(w_{c}\right)\left\{\theta\left(w_{k}^{2}-w_{c}^{2}\right) \delta \psi(x)+\delta\left(w_{k}^{2}-w_{c}^{2}\right)\right. \\
& \left.\times\left[\delta \psi(x)-\delta \psi_{m}\right]\right\},
\end{aligned}
$$

where the substitution (A5) is assumed. The same terms as functions of $(x, w)$ are determined by the formulas

$$
\begin{gathered}
\delta f_{k, t}^{i n j}(x, w)=\delta f_{k}(x, w) \theta\left( \pm w-w_{*}(x)\right), \\
\delta f_{k, r}^{i n j}(x, w)=\delta f_{k}(x, w) \theta\left(w_{*}^{2}(x)-w^{2}\right),
\end{gathered}
$$

and

$$
\begin{aligned}
\delta f_{k, t}^{i n d}(x, w)= & \frac{1}{\sqrt{\pi}} e^{-w^{2}+\psi(x)-\psi_{k}}\left\{\theta\left( \pm w-w_{*}(x)\right) \delta \psi(x)\right. \\
& \left.\mp \frac{1}{2 w} \delta\left( \pm w-w_{*}(x)\right)\left[\delta \psi(x)-\delta \psi_{m}\right]\right\},
\end{aligned}
$$

$$
\begin{aligned}
\delta f_{k, r}^{i n d}(x, w)= & \frac{1}{\sqrt{\pi}} e^{-w^{2}+\psi(x)-\psi_{k}\left\{\theta\left(w_{*}^{2}(x)-w^{2}\right) \delta \psi(x)\right.} \\
& \left.+\delta\left(w_{*}^{2}(x)-w^{2}\right)\left[\delta \psi(x)-\delta \psi_{m}\right]\right\} .
\end{aligned}
$$

Apparently, $\delta f^{i n j}$ has a meaning of the distribution function of randomly injected electrons, while $\delta f^{\text {ind }}$ describes the change in the steady-state distribution induced by injected electrons. 
The obtained fluctuations of the distribution function allows one to compute each contribution to the fluctuations of the electron density $\delta n(x)$ by integrating over velocities. Changing the integration over $w$ to that over the contact injection velocities $w_{c}$, we find

$$
\begin{aligned}
\delta n_{k, j}(x) & =\int_{-\infty}^{\infty} \delta f_{k, j}(x, w) d w \\
& =\int_{-\infty}^{\infty} \frac{\delta f_{k, j}\left(w_{c}\right) w_{c} d w_{c}}{\operatorname{sgn}\left(w_{c}\right) \sqrt{w_{c}^{2}+\psi(x)-\psi_{k}}} .
\end{aligned}
$$

Thus, by using Eqs. (A10) and (A11), one obtains for the injected density fluctuations

$$
\begin{gathered}
\delta n_{L, t}^{i n j}(x)=\int_{w_{L}}^{\infty} \frac{\delta f_{L}\left(w_{c}\right) w_{c} d w_{c}}{\sqrt{w_{c}^{2}+\psi(x)-\psi_{L}}}, \\
\delta n_{R, t}^{i n j}(x)=-\int_{-\infty}^{-w_{R}} \frac{\delta f_{R}\left(w_{c}\right) w_{c} d w_{c}}{\sqrt{w_{c}^{2}+\psi(x)-\psi_{R}}},
\end{gathered}
$$

$$
\delta n_{k, r}^{i n j}(x)=2 \int_{\sqrt{\psi_{k}-\psi(x)}}^{w_{k}} \frac{\delta f_{k}\left(w_{c}\right) w_{c} d w_{c}}{\sqrt{w_{c}^{2}+\psi(x)-\psi_{k}}},
$$

and for the induced fluctuations

$$
\begin{aligned}
& \delta n_{k, t}^{i n d}(x)=n_{k, t} \delta \psi(x)-\frac{e^{-\psi_{k}-\psi_{m}}}{2 \sqrt{\pi} w_{*}(x)}\left[\delta \psi(x)-\delta \psi_{m}\right], \\
& \delta n_{k, r}^{\text {ind }}(x)=n_{k, r} \delta \psi(x)+\frac{e^{-\psi_{k}-\psi_{m}}}{\sqrt{\pi} w_{*}(x)}\left[\delta \psi(x)-\delta \psi_{m}\right] .
\end{aligned}
$$

Here, the contributions (A15) can be interpreted as the electron-density fluctuations at a slice $x$ caused by the stochastic injection from the contacts to the base. The contributions (A16) are related to a variation of the stationary electron density due to a local variation of the potential and its minimal value (a self-consistent response). As before, the terms $\delta n_{L, r}$ and $\delta n_{R, r}$ are defined on the intervals $0<x$ $<x_{m}$ and $x_{m}<x<\lambda$, respectively, while the terms $\delta n_{k, t}$ are defined on the whole range $0<x<\lambda$.
${ }^{1}$ M. J. M. de Jong and C. W. J. Beenakker, in Mesoscopic Electron Transport, edited by L. P. Kowenhoven, G. Schön, and L. L. Sohn (Kluwer, Dordrecht, 1997), p. 225.

${ }^{2}$ W. Schottky, Ann. Phys. (Leipzig) 57, 541 (1918).

${ }^{3}$ R. Landauer and Th. Martin, Physica B 175, 167 (1991); R. Landauer, Phys. Rev. B 47, 16427 (1993); Physica B 227, 156 (1996); Nature (London) 392, 658 (1998).

${ }^{4} \mathrm{M}$. Büttiker, in 14th International Conference on Noise in Physical Systems and 1/f Fluctuations, edited by V. Bareikis and R. Katilius (World Scientific, Singapore, 1995), p. 35; J. Math. Phys. 37, 4793 (1996).

${ }^{5}$ T. González, O. M. Bulashenko, J. Mateos, D. Pardo, and L. Reggiani, Phys. Rev. B 56, 6424 (1997).

${ }^{6}$ O. M. Bulashenko, J. Mateos, D. Pardo, T. González, L. Reggiani, and J. M. Rubí, Phys. Rev. B 57, 1366 (1998).

${ }^{7}$ A. J. Rack, Bell Syst. Tech. J. 17, 592 (1938).

${ }^{8}$ D. O. North, RCA Rev. 4, 441 (1940). This paper is a part of a series of publications by B. J. Thompson, D. O. North, and W. A. Harris in RCA Review during 1940-41, under the common title "Fluctuations in space-charge-limited currents at moderately high frequencies.'

${ }^{9}$ A. van der Ziel, Noise (Prentice-Hall, Englewood Cliffs, NJ, 1954), Chaps. 5-1 and 14-2.

${ }^{10}$ Y. P. Li, D. C. Tsui, J. J. Heremans, J. A. Simmons, and G. W. Weimann, Appl. Phys. Lett. 57, 774 (1990).

${ }^{11}$ E. R. Brown, IEEE Trans. Electron Devices 39, 2686 (1992).

${ }^{12}$ H. C. Liu, J. Li, G. C. Aers, C. R. Leavens, M. Buchanan, and Z. R. Wasilewski, Phys. Rev. B 51, 5116 (1995).

${ }^{13}$ G. Iannaccone, G. Lombardi, M. Macucci, and B. Pellegrini, Phys. Rev. Lett. 80, 1054 (1998).

${ }^{14}$ Ya. M. Blanter and M. Büttiker, Phys. Rev. B 59, 10217 (1999).

${ }^{15}$ A. N. Korotkov, D. V. Averin, K. K. Likharev, and S. A. Vasenko, in Single-Electron Tunneling and Mesoscopic Devices, edited by H. Koch and H. Lübbig (Springer, Berlin, 1992), p. 15.

${ }^{16}$ S. Hershfield, J. H. Davies, P. Hyldgaard, C. J. Stanton, and J. W. Wilkins, Phys. Rev. B 47, 1967 (1993).

${ }^{17}$ U. Hanke, Yu. M. Galperin, K. A. Chao, and N. Zou, Phys. Rev. B 48, 17209 (1993).

${ }^{18}$ A. Imamoḡlu and Y. Yamamoto, Phys. Rev. Lett. 70, 3327 (1993).

${ }^{19}$ M. Yamanishi, K. Watanabe, N. Jikutani, and M. Ueda, Phys. Rev. Lett. 76, 3432 (1996).

${ }^{20}$ K. A. Matsuoka and K. K. Likharev, Phys. Rev. B 57, 15613 (1998).

${ }^{21}$ H. Birk, M. J. M. de Jong, and C. Schönenberger, Phys. Rev. Lett. 75, 1610 (1995).

${ }^{22}$ Y. Naveh, D. V. Averin, and K. K. Likharev, Phys. Rev. Lett. 79, 3482 (1997); Phys. Rev. B 59, 2848 (1999).

${ }^{23}$ K. E. Nagaev, Phys. Rev. B 57, 4628 (1998).

${ }^{24}$ Y. Naveh, A. N. Korotkov, and K. K. Likharev, Phys. Rev. B 60, R2169 (1999).

${ }^{25}$ T. González, C. González, J. Mateos, D. Pardo, L. Reggiani, O. M. Bulashenko, and J. M. Rubí, Phys. Rev. Lett. 80, 2901 (1998); T. González, J. Mateos, D. Pardo, O. M. Bulashenko, and L. Reggiani, Phys. Rev. B 60, 2670 (1999).

${ }^{26}$ C. W. J. Beenakker, Phys. Rev. Lett. 82, 2761 (1999).

${ }^{27}$ H. Schomerus, E. G. Mishchenko, and C. W. J. Beenakker, Phys. Rev. B 60, 5839 (1999).

${ }^{28}$ P. K. Tien and J. Moshman, J. Appl. Phys. 27, 1067 (1956).

${ }^{29}$ C. P. Wen and J. E. Rowe, IEEE Trans. Electron Devices 11, 90 (1964).

${ }^{30}$ S. Saito, Y. Fujii, and A. Iwamoto, IEEE Trans. Electron Devices 19, 1190 (1972).

${ }^{31}$ The closest analog from vacuum electronics is a double-cathode valve (Ref. 32) containing two hot cathodes under the same temperature opposite each other and operating under a space- 
charge-limited transport regime; see also Ref. 33.

${ }^{32}$ K. S. Knol and G. Diemer, Philips Res. Rep. 5, 131 (1950).

${ }^{33}$ O. M. Bulashenko, J. M. Rubí, and V. A. Kochelap, Appl. Phys. Lett. 75, 2614 (1999).

${ }^{34}$ M. Reznikov, M. Heiblum, H. Shtrikman, and D. Mahalu, Phys. Rev. Lett. 75, 3340 (1995).

${ }^{35}$ A. Kumar, L. Saminadayar, D. C. Glattli, Y. Jin, and B. Etienne, Phys. Rev. Lett. 76, 2778 (1996).

${ }^{36}$ R. J. Schoelkopf, P. J. Burke, A. A. Kozhevnikov, D. E. Prober, and M. J. Rooks, Phys. Rev. Lett. 78, 3370 (1997).

${ }^{37}$ M. A. Lampert and P. Mark, Current Injection in Solids (Academic, New York, 1970).

${ }^{38}$ V. V. Mitin, V. A. Kochelap, and M. A. Stroscio, Quantum Heterostructures (Cambridge University Press, New York, 1999).

${ }^{39}$ C. W. J. Beenakker and H. van Houten, in Solid State Physics: Advances in Research and Applications, edited by H. Ehrenreich and D. Turnbull (Academic, San Diego, 1991), Vol. 44.

${ }^{40}$ G. R. Facer, B. E. Kane, A. S. Dzurak, R. J. Heron, N. E. Lumpkin, R. G. Clark, L. N. Pfeiffer, and K. W. West, Phys. Rev. B 59, 4622 (1999).

${ }^{41}$ It follows from the fact that the Coulomb potential is a power function having no characteristic scale. The distance between the contacts may be chosen as the length scale for estimates.

${ }^{42}$ A. A. Vlasov, Zh. Eksp. Teor. Fiz. 8, 291 (1938).

${ }^{43}$ R. Balescu, Equilibrium and Nonequilibrium Statistical Mechanics (Wiley, New York, 1975), Chap. 11.7.

${ }^{44}$ Sh. Kogan, Electronic Noise and Fluctuations in Solids (Cam- bridge University Press, Cambridge, 1996), Chap. 3.6.

${ }^{45}$ T. C. Fry, Phys. Rev. 17, 441 (1921).

${ }^{46}$ I. Langmuir, Phys. Rev. 21, 419 (1923).

${ }^{47}$ A. van der Ziel, Solid State Physical Electronics, 3rd ed. (Prentice-Hall, Englewood Cliffs, NJ, 1976), Chap. 8-1.

${ }^{48}$ T. González, J. Mateos, D. Pardo, O. M. Bulashenko, and L. Reggiani, Semicond. Sci. Technol. 13, 714 (1998).

${ }^{49}$ O. M. Bulashenko, J. M. Rubí, and V. A. Kochelap, cond-mat/9911395 (unpublished).

${ }^{50}$ J. R. Hayes, A. F. J. Levi, and W. Wiegmann, Phys. Rev. Lett. 54, 1570 (1985).

${ }^{51}$ M. Heiblum, M. I. Nathan, D. C. Thomas, and C. M. Knoedler, Phys. Rev. Lett. 55, 2200 (1985).

${ }^{52}$ O. M. Bulashenko, G. Gomila, J. M. Rubí, and V. A. Kochelap, Appl. Phys. Lett. 70, 3248 (1997); J. Appl. Phys. 83, 2610 (1998).

${ }^{53}$ An electron with $\varepsilon=V_{m}$ comes to rest in the virtual cathode, producing an infinitely large perturbation of the current. However, this effect is unphysical, since that point corresponds to an unstable equilibrium for the electron. The physically meaningful quantity is the current noise obtained by the integration over all injection energies, and that integral is well defined because of the logarithmic type of the singularity.

${ }^{54}$ J. Yao, C. J. Chen, K. K. Choi, W. H. Chang, and D. C. Tsui, Appl. Phys. Lett. 72, 453 (1998).

${ }^{55}$ B. E. A. Saleh and M. C. Teich, Proc. IEEE 80, 451 (1992). 